\title{
CORRECTED RANDOM WALK APPROXIMATIONS TO FREE BOUNDARY PROBLEMS IN OPTIMAL STOPPING
}

\author{
TZE LEUNG LAI, ${ }^{*}$ Stanford University \\ YI-CHING YAO,** Academia Sinica \\ FARID AITSAHLIA, ${ }^{* * *}$ University of Florida \\ Dedicated to Kai Lai Chung on the Occasion of his 90th Birthday
}

\begin{abstract}
Corrected random walk approximations to continuous-time optimal stopping boundaries for Brownian motion, first introduced by Chernoff and Petkau, have provided powerful computational tools in option pricing and sequential analysis. This paper develops the theory of these second-order approximations and describes some new applications.
\end{abstract}

Keywords: Optimal stopping; free boundary partial differential equation; random walk; renewal theory; singular stochastic control

2000 Mathematics Subject Classification: Primary 60G40

Secondary 60H30; 90C39

\section{Introduction}

Chernoff [11] and Chernoff and Petkau [13] introduced a second-order correction for computing the optimal stopping boundary of a Brownian motion via a random walk approximation and the backward induction algorithm of discrete-time dynamic programming. This correction is based on the solution to an associated stopping problem in which the stopping time $0 \geq \tau \geq t$ for a Brownian motion $\left\{B_{t}, t \leq 0\right\}$ is chosen to maximize $\mathrm{E}\left[g\left(B_{\tau}, \tau\right) \mid B_{t}=y_{0}\right]$, where $y_{0}<0$,

$$
g(y, s)=-s \mathbf{1}_{\{s<0\}}+y^{2} \mathbf{1}_{\{s=0, y \leq 0\}},
$$

and $\mathbf{1}_{\{\cdot\}}$ denotes the indicator function. Approximating Brownian motion by a zero-mean random walk so that the discrete-time optimal stopping problem maximizes $\mathrm{E}\left[g\left(y_{0}+\sqrt{\delta} S_{N}\right.\right.$, $t+\delta N)$ ] over integer-valued stopping times $N$, Hogan [32] generalized the previous work of Chernoff [11] for normal random walks and Chernoff and Petkau [13] for Bernoulli random walks, and showed that the (discrete-time) optimal stopping boundary $b_{\delta}(t)$ for the approximating random walk is related to the (continuous-time) optimal stopping boundary $b(t)(\equiv 0)$ in the associated problem for Brownian motion by

$$
b_{\delta}(t)=b(t)-\rho \sqrt{\delta}+o(\sqrt{\delta}),
$$

\footnotetext{
Received 23 June 2005; revision received 11 June 2007.

* Postal address: Department of Statistics, Stanford University, Stanford, CA 94305, USA.

Email address: lait@stat.stanford.edu.

** Postal address: Institute of Statistical Science, Academia Sinica, Taipei 115, Taiwan, ROC.

Email address: yao@stat.sinica.edu.tw.

*** Postal address: Department of Industrial and Systems Engineering, University of Florida, PO Box 116595, Gainesville, FL 32611-6595, USA. Email address: farid@ise.ufl.edu.
} 
where $\rho=\mathrm{E} S_{\tau_{+}}^{2} / 2 \mathrm{E} S_{\tau_{+}}, \tau_{+}=\inf \left\{n: S_{n}>0\right\}$, and $S_{n}=\sum_{i=1}^{n} X_{i}$ with $\mathrm{E} X_{i}=0$ and $\mathrm{E} X_{i}^{2}=1$. Actually, Hogan only proved that $\lim _{n \rightarrow \infty} b_{1}(-n)=-\rho$. However, this result corresponding to the special case in which $\delta=1$ can be combined with the scaling property $g(\sqrt{\delta} y, \delta s)=\delta g(y, s)$ of (1.1) to yield (1.2) for all negative $t$ bounded away from 0 . Whereas (1.2) has been derived only for the special payoff function (1.1), Chernoff [12] gave some heuristic argument based on Taylor expansions around the optimal stopping boundary to justify the use of (1.2) for more general payoff functions. In this paper, by making use of results from a canonical optimal stopping problem (see Section 3.2) that generalizes Chernoff's 'associated stopping problem', we derive (1.2) for general payoff functions that satisfy regularity conditions of the type given in [48] and [49].

Besides sequential analysis problems that motivated Chernoff [11] and Chernoff and Petkau [15] to develop corrected random walk approximations to optimal stopping boundaries for Brownian motion, we were also motivated by certain computational problems in singular stochastic control (see Section 5) and option pricing. For an illustration of the importance of the continuity correction of the binomial approximation of the optimal exercise boundary of an American option, we refer to the extensive numerical study of AitSahlia and Lai [1]. In the Black-Scholes theory, the price of a standard American option is $V:=$ $\sup _{\tau \in \mathcal{T}\left(0, T^{*}\right)} \mathrm{E}\left[\mathrm{e}^{-r \tau} f\left(P_{\tau}\right)\right]$, where $f(P)=(K-P)^{+}$or $(P-K)^{+}$for a put or call option, with strike price $K$ and expiration date $T^{*}, \mathcal{T}(a, b)$ is the set of stopping times taking values between $a$ and $b$ with $b>a$, and $P_{t}:=P_{0} \exp \left\{\left(r-d-\sigma^{2} / 2\right) t+\sigma B_{t}\right\}$ is the price (under the risk-neutral measure) of the risky asset at time $t$, in which $r$ is the risk-free interest rate, $d$ is the dividend rate, and $\sigma$ is the volatility. A standard method to compute American option prices, introduced by Cox et al. [18], is to approximate the geometric Brownian motion $P_{t}$ by a binomial tree, for which backward induction can be applied to find the value $V_{n}$ in the corresponding discrete optimal stopping problem with $n$ evenly spaced time steps. When $d=0$ and the binomial tree approximating $P_{t}$ corresponds to a symmetric Bernoulli walk approximating $B_{t}$, Lamberton [39] established the error bounds $-c_{1} n^{-2 / 3} \leq V_{n}-V \leq c_{2} n^{-3 / 4}$ for some positive constants $c_{1}$ and $c_{2}$ (depending on the initial price $P_{0}$ ), which was later, in [40], extended to $d \geq 0$ and improved to

$$
\left|V_{n}-V\right| \leq c\left(\frac{\sqrt{\log n}}{n}\right)^{4 / 5}
$$

for some positive constant $c$ (depending on $P_{0}$ ). Lamberton also indicated that a major reason why sharper bounds could not be established is that $\partial^{2} V / \partial P^{2}$ and the derivative of the optimal stopping boundary become infinite when approaching the expiration date $T^{*}$. AitSahlia and Lai [1] circumvented this difficulty by using another method to compute the stopping boundary and the value function near $T^{*}$. This method involves numerical solution of the integral equation defining the optimal stopping boundary $b(t)$ (see (2.11), below), which is reasonably fast when $t$ is near $T^{*}$ although its computation becomes increasingly expensive as $t$ moves further from $T^{*}$. Moreover, instead of binomial trees to approximate $P_{t}$, AitSahlia and Lai used symmetric Bernoulli walks to approximate $B_{t}$ in computing the stopping boundary and value function on $\left[0, T^{*}-\varepsilon\right]$ for some small $\varepsilon>0$, on which both functions are smooth and (1.2) holds; see Theorem 3.1, below.

In Section 3 we prove (1.2) for general payoff functions by using an induction argument that proceeds backwards in time, initializing at time $T<T^{*}$ with approximations that are within $O(\delta)$ of the value function $v(x, T)$ and $O(\sqrt{\delta})$ of the optimal stopping boundary $b(T)$ at $T$. The induction argument also establishes that $v_{\delta}\left(x, t_{i}\right)-v\left(x, t_{i}\right)=O(\delta)$ uniformly in $t_{i}$, where $v$ is the value function of the optimally stopped Brownian motion and $v_{\delta}$ is the value function 
of the discrete-time optimal stopping problem in which stopping can only occur at times $t_{i}$ with $t_{i-1}-t_{i}=\delta$. Applying these results to American option pricing with $\delta=1 / n$ yields $\widetilde{V}_{n}-V=$ $O\left(n^{-1}\right)$ as an improvement of (1.3), where $\widetilde{V}_{n}$ is the value function for the approximating Bernoulli random walk suitably initialized at $T=T^{*}-\varepsilon$. (See Remark 3.3, below, for further discussion.) The proof of the main theorem in Section 3.3 requires renewal theory for random walks related to excess over the boundary and certain basic properties of the value functions and stopping boundaries of optimal stopping problems for Brownian motion. Section 2 reviews some of these properties and proves a basic decomposition formula which we use to derive other basic properties and computational methods in Sections 3.3 and 3.4. In particular, Section 2.1 reviews the partial differential equation (PDE) literature on the differentiability of the optimal stopping boundary $b(t)$, which has been implicitly assumed by Chernoff [11], [12] in his heuristic derivation of (1.2) for general payoff problems.

For standard normal $X_{i}$ and constant boundary $b(t) \equiv b$, the boundary correction (1.2) has also been introduced by Broadie et al. [7] to approximate the value of a discretely monitored European barrier option by its continuously monitored counterpart whose value, denoted $V(H)$, is given by an explicit formula that depends on the barrier $H$, volatility $\sigma$, strike price $K$, expiration date $T^{*}$, as well as the type of barrier (up or down) and the type of option (knock-in or knock-out). When the barrier option is monitored only at times $i \delta(i=0,1, \ldots, n)$, with $\delta=T^{*} / n$, Broadie et al. [7] proposed to use a continuity correction for the barrier, similar to (1.2), to approximate the price $V_{n}(H)$ of the discretely monitored option with barrier $H$ by the explicit formula of the corresponding continuously monitored option price, and showed that

$$
V_{n}(H)=V\left(H \exp \left( \pm \rho \sigma \sqrt{\frac{T^{*}}{n}}\right)\right)+o\left(n^{-1 / 2}\right),
$$

where ' + ' and ' - ' applies to the up and down barrier, respectively, and $\rho\left(=\mathrm{E} S_{\tau_{+}}^{2} / 2 \mathrm{E} S_{\tau_{+}}\right) \approx$ 0.5826 for normal $X_{i}$. Note that whereas (1.3) is concerned with approximating the value of an optimal stopping problem for Brownian motion by that of a zero-mean random walk, (1.4) considers the reverse approximation that approximates the stopped value of a random walk by that of Brownian motion. In Section 4 we apply (1.2) in this reverse manner to two classical optimal stopping problems. In Section 5 we consider another application of (1.2), namely evaluating the optimal (reflection) boundary in singular stochastic control problems for Brownian motion via corrected random walk approximations.

\section{Value function and optimal stopping boundary}

Instead of (1.1), we shall consider more general payoff (reward) functions $g(x, t)$ that satisfy certain regularity conditions. The corresponding value function is

$$
v(x, t)=\sup _{\tau \in \mathcal{T}\left(t, T^{*}\right)} \mathrm{E}\left[g\left(B_{\tau}, \tau\right) \mid B_{t}=x\right],
$$

where $\widetilde{T} \leq t<T^{*}$, in which $g(x, t)$ has a left-hand limit $g\left(x, T^{*}-\right)$ as $t \uparrow T^{*}$. Let $g_{*}(x)=$ $g\left(x, T^{*}\right)-g\left(x, T^{*}-\right)$. Whereas the optimal stopping rule for (1.1) stops sampling as soon as $B_{t} \geq 0$ (so $b(t) \equiv 0$ is the optimal stopping boundary), the optimal stopping rule in the general case has continuation region $\mathcal{R}:=\{(x, t): v(x, t)>g(x, t)\}$, which need not be a connected set. To extend (1.2) to the present case, we therefore assume that

$$
\mathcal{R}=\left\{(x, t): x<b(t), \widetilde{T} \leq t<T^{*}\right\},
$$


where the boundary $b(t)$ converges to a limit $b\left(T^{*}\right)$ as $t \uparrow T^{*}$ and satisfies the smoothness condition (2.4), below. While Theorem 3.1 in Section 3 shows that (1.2) holds for continuation regions of the form (2.2), a similar correction with $-\rho \sqrt{\delta}$ in (1.2) replaced by $\rho \sqrt{\delta}$ is also applicable to continuation regions of the form $\{(x, t): x>b(t)\}$, and we can combine the corrections for the upper and lower boundaries when the continuation region is of the form $\left\{(x, t): b_{1}(t)<x<b_{2}(t)\right\}$.

Let $\mathcal{R}_{\xi}=\left\{(x, t): x<b(t)+\xi, \widetilde{T} \leq t<T^{*}\right\}\left(\mathcal{R}_{0}=\mathcal{R}\right)$. Because our derivation of (1.2) relies on Taylor expansions, we shall assume that $g$ belongs to the class $C^{2,1}$ on $\mathbb{R} \times\left[\widetilde{T}, T^{*}\right)$ and $C^{3,2}$ on $\mathcal{R}_{\xi}$ such that

$$
g, g_{x}, g_{x x}, g_{x x x}, g_{t}, g_{t t}, g_{x t}, g_{*}, g_{*}^{\prime}, g_{*}^{\prime \prime}, g_{*}^{\prime \prime \prime} \quad \text { are bounded by } \mathrm{e}^{\beta(x)} \text { on } \mathcal{R}_{\xi},
$$

for some $\xi>0$, where $\beta(x)$ satisfies the growth condition $\beta(x)=O(|x|)$ as $x \rightarrow-\infty$, which is somewhat stronger than van Moerbeke's Tychonov condition [48, p. 115].

We assume, furthermore, that

$$
b^{\prime}(t) \text { is continuous at every } t \in\left[\widetilde{T}, T^{*}\right),
$$

so that we can also apply Taylor's theorem to the stopping boundary. Under these assumptions, we have the following result due to van Moerbeke [48], [49].

Lemma 2.1. Let $G(x, t)=\left(\partial / \partial t+\frac{1}{2} \partial^{2} / \partial x^{2}\right) g(x, t)$.

(a) If $G(x, t)>0$ or $G(x, t)=0$ and $G_{x}(x, t) \neq 0$, then $(x, t)$ is a continuation point.

(b) The functions $(\partial / \partial x)(v-g)$ and $(\partial / \partial t)(v-g)$ are continuous across the stopping boundary $b(\cdot)$ and vanish at $x=b(t)$ for $t \in\left[\widetilde{T}, T^{*}\right)$.

(c) The function $\frac{1}{2}\left(\partial^{2} / \partial x^{2}\right)(v-g)$ is continuous in the continuation region $\mathcal{R}$ up to the boundary $b(\cdot)$ and is equal to $-G$ at $x=b(t)$ for $t \in\left[\widetilde{T}, T^{*}\right)$.

(d) The function $\left(\partial^{2} / \partial x \partial t\right)(v-g)$ is continuous in $\mathcal{R}$ up to the boundary $b(\cdot)$ and is equal to $2 b^{\prime}(t) G(b(t), t)$ at $x=b(t)$ for $t \in\left[\widetilde{T}, T^{*}\right) ; v \in C^{\infty}(\mathcal{R})$ and $(\partial / \partial t+$ $\left.\frac{1}{2} \partial^{2} / \partial x^{2}\right) v(x, t)=0$ for $(x, t) \in \mathcal{R}$.

(e) We have $\left(\partial^{2} / \partial t^{2}\right)(v-g)(b(t), t)=-2 G(b(t), t)\left(b^{\prime}(t)\right)^{2}$ for $t \in\left[\widetilde{T}, T^{*}\right)$.

Making use of [48, Lemma 4], and (2.4) together with Lemma 2.1(e), we next prove the following important property of $\partial^{5} v / \partial x^{5}$ that will be used in Section 3 .

Lemma 2.2. Assume, furthermore, that

$$
g_{t t} \in C^{2,1}, \quad g_{x x} \in C^{1,1} \quad \text { on } \mathcal{R}_{\xi} \text {, for some } \xi>0 .
$$

Then $\partial^{5} v / \partial x^{5}$ is continuous in the continuation region $\mathcal{R}$ up to the boundary $b(\cdot)$.

Proof. Define $u(x, t)=v_{t t}(x, t)-g_{t t}(x, t)+2\left(b^{\prime}(t)\right)^{2} G(b(t), t)$ on $\mathcal{R}$. By Lemma 2.1(e), $u(b(t), t)=0$. We shall show that

$$
\left(b^{\prime}(t)\right)^{2} G(b(t), t) \quad \text { is continuously differentiable for } \widetilde{T} \leq t<T^{*} .
$$

Combining this result with (2.5) shows that $(\partial u / \partial t)+\frac{1}{2}\left(\partial^{2} u / \partial x^{2}\right)$ is continuous in $\mathcal{R}$. Hence, by [48, Lemma 4], $\partial u / \partial x$ is continuous in $\mathcal{R}$ up to $b(\cdot)$. In view of (2.5), this implies that 
$\partial v_{t t} / \partial x$ is continuous in $\mathcal{R}$ up to $b(\cdot)$. Since $v_{t}=-\frac{1}{2} v_{x x}$ implies that $v_{t t}=\frac{1}{4} \partial^{4} v / \partial x^{4}$, it then follows that $\partial v_{t t} / \partial x=\frac{1}{4} \partial^{5} v / \partial x^{5}$ is continuous in $\mathcal{R}$ up to $b(\cdot)$.

To prove (2.6), we apply the integral equation for $f(t):=-\left(b^{\prime}(t)\right)^{2} G(b(t), t)$, similar to [49, Equation (37)], which is derived by making use of Lemma 2.1(e) to arrive at a free boundary problem for $v_{t t}-g_{t t}$ and then applying Green's theorem. Specifically, for $T^{\prime} \in\left[\widetilde{T}, T^{*}\right)$ and $\widetilde{T} \leq t \leq T^{\prime}$

$$
\begin{aligned}
f(t)= & \int_{0}^{T^{\prime}-t}\left\{\int_{-\infty}^{b\left(T^{\prime}-\tau\right)} \psi(b(t) ; y, \tau) \frac{\partial}{\partial t} G\left(y, T^{\prime}-\tau\right) \mathrm{d} y\right\} \frac{\mathrm{d} \tau}{\sqrt{T^{\prime}-t-\tau}} \\
& +\int_{0}^{T^{\prime}-t} \frac{\psi\left(b(t) ; b\left(T^{\prime}-\tau\right), \tau\right)}{\sqrt{T^{\prime}-t-\tau}} f\left(T^{\prime}-\tau\right) \mathrm{d} \tau \\
& -\int_{-\infty}^{b\left(T^{\prime}\right)} \frac{\psi(b(t) ; y, 0)}{\sqrt{T^{\prime}-t}}\left\{\frac{1}{2}\left(v_{t t}-g_{t t}\right)\left(y, T^{\prime}\right)\right\} \mathrm{d} y,
\end{aligned}
$$

where $\psi(x ; y, \tau)=(\partial / \partial x) \phi\left((x-y) / \sqrt{T^{\prime}-t-\tau}\right)$ and $\phi$ is the standard normal density function. Since, by $(2.4), b(t)$ is continuously differentiable on $\left[\widetilde{T}, T^{\prime}\right]$, so is $f(t)$.

\subsection{Smoothness of the optimal stopping boundary}

Condition (2.4) requires that the optimal stopping boundary be continuously differentiable, but the issue of differentiability of the optimal stopping boundary has been mostly neglected in the probability literature. In contrast, the PDE literature contains extensive studies of various free boundary problems involving parabolic PDEs; see, for example, [26] and [29] on the theory of variational or quasi-variational inequalities and the existence, uniqueness, and regularity of the solution and smoothness of the free boundary. Friedman [25], [27], [28] has applied the theory to a number of problems in optimal stopping, sequential analysis, and stochastic control.

For American put options, the issue of differentiability of the optimal stopping (early exercise) boundary has recently been taken up in the literature. By referring to McKean [42] and van Moerbeke [49], Myneni [43] stated that the early exercise boundary is $C^{1}$. However, Peskir [44] and Peskir and Shiryaev [45] recently pointed out that neither [42] nor [49] contains a proof of this result. Conversely, using parabolic PDE techniques involving variational inequality theory, Chen and Chadam [9] have proved the existence and uniqueness of the solution to the free boundary PDE. Moreover, they have shown that the early exercise boundary, as the unique solution of an integral equation, is in fact $C^{\infty}$. Further results on convexity properties of the option price and early exercise boundary can be found in [10], [20], and [21].

\subsection{Integral representations and Doob-Meyer decomposition of value functions}

The following theorem provides a representation of $v$ in terms of the optimal stopping boundary and plays an important role in our subsequent development. Let $\mathrm{E}_{x, t}$ denote the conditional expectation given $B_{t}=x$.

Theorem 2.1. For $\widetilde{T} \leq t \leq T<T^{*}$,

$$
v(x, t)=\mathrm{E}_{x, t} v\left(B_{T}, T\right)-\int_{t}^{T} \mathrm{E}_{x, t}\left[G\left(B_{s}, s\right) \mathbf{1}_{\left\{B_{s} \geq b(s)\right\}}\right] \mathrm{d} s .
$$

The process $\left\{v\left(B_{t}, t\right), \mathcal{F}_{t}, \widetilde{T} \leq t \leq T\right\}$ is the minimal right-continuous supermartingale that majorizes $\left\{g\left(B_{t}, t\right), \widetilde{T} \leq t \leq T\right)$, where $\left\{\mathcal{F}_{t}, \widetilde{T} \leq t \leq T\right\}$ is a filtration to which $\left\{B_{t}, \widetilde{T} \leq t \leq T\right\}$ is adapted; see [23, Theorem 2]. Therefore, the Doob-Meyer decomposition 
yields

$$
v\left(B_{t}, t\right)=M_{t}-A_{t},
$$

where $M_{t}$ is a martingale and $A_{t}$ is a predictable nondecreasing process, and the choice of $M_{t}-M_{\widetilde{T}}$ and $A_{t}-A_{\widetilde{T}}$ in (2.8) is essentially unique. To find $M$ and $A$, we apply the generalized Ito formula [36, Theorem 2.10.1] to $v\left(B_{t}, t\right)$. Since $v$ is $C^{1}$ (in view of Lemma 2.1(b)) and piecewise $C^{2,1}$ on $\mathbb{R} \times[\widetilde{T}, T]$, the generalized Ito formula yields, for $T>t$,

$$
v\left(B_{T}, T\right)-v\left(B_{t}, t\right)=\int_{t}^{T}\left(v_{t}+\frac{v_{x x}}{2}\right)\left(B_{s}, s\right) \mathrm{d} s+\int_{t}^{T} v_{x}\left(B_{s}, s\right) \mathrm{d} B_{s},
$$

which gives the martingale component $\int_{t}^{T} v_{x}\left(B_{s}, s\right) \mathrm{d} B_{s}$ in the Doob-Meyer decomposition (2.8). Since $\left(v_{t}+v_{x x} / 2\right)(x, t)=0$ if $x<b(t)$, and $=G(x, t)$ if $x>b(t)$, taking expectation conditional on $B_{t}=x$ in (2.9) gives the conclusion (2.7) of Theorem 2.1. For similar arguments in the special case of American options, see [33, Section 4] and [35, Section 2.7].

If $g\left(x, T^{*}-\right) \leq g\left(x, T^{*}\right)$ for all $x$ (i.e. $g_{*}(x) \geq 0$ for all $x$ ), then $\lim _{T \uparrow T^{*} v(x, T)=}$ $v\left(x, T^{*}\right)=g\left(x, T^{*}\right)$, so letting $T \uparrow T^{*}$ in (2.7) yields

$$
v(x, t)=\mathrm{E}_{x, t} g\left(B_{T^{*}}, T^{*}\right)-\int_{t}^{T^{*}} \mathrm{E}\left[G(x+\sqrt{s-t} Z, s) \mathbf{1}_{\{x+\sqrt{s-t} Z \geq b(s)\}}\right] \mathrm{d} s,
$$

where $Z$ is a standard normal random variable. In particular, for American options, (2.10) corresponds to the decomposition formula due to Jacka [33] and Carr et al. [8]

American option price $=$ European option price + Early exercise premium,

in which the integrand in the integral defining the early exercise premium is an explicit function of the optimal stopping boundary, noting that $-G \geq 0$ at stopping points by Lemma 2.1(a). Setting $x=b(t)$ in (2.10) leads to the following integral equation defining $b$ :

$g(b(t), t)=\mathrm{E} g\left(b(t)+\sqrt{T^{*}-t} Z, T^{*}\right)-\int_{t}^{T^{*}} \mathrm{E}\left[G(b(t)+\sqrt{s-t} Z, s) \mathbf{1}_{\{\sqrt{s-t} Z \geq b(s)-b(t)\}}\right] \mathrm{d} s$.

AitSahlia and Lai [1] showed how (2.10) and (2.11) can be used to develop a relatively fast and accurate method to compute $v(x, t)$ and $b(t)$ for $t$ near the expiration date $T^{*}$ in the case of American options, thereby circumventing difficulties in the Bernoulli walk approximation due to unbounded $\partial^{2} v / \partial x^{2}$ and $b^{\prime}$ as $t \uparrow T^{*}$. A similar idea was used by Brezzi and Lai [6, p. 92] to avoid the singularity at $T^{*}$ in initializing the Bernoulli walk method to compute the optimal stopping boundary for bandit problems. In Section 3.3 we give another application of (2.10) to bound the fifth derivative of $v$ (with respect to $x$ ), which we use to prove (1.2) for general payoff functions $g$.

An alternative derivation of (2.11) by Evans et al. [22] for the special case of American options is to use Green's function $\gamma(z, s ; \xi, u)=(u-s)^{-1 / 2} \phi((z-\xi) / \sqrt{u-s}), u>s$, and apply Green's theorem. In fact, this approach involving Green's function and Green's theorem leads to a variety of integral equations for the optimal stopping boundary; see [9].

\section{Theory of corrected random walk approximations}

In this section we first present a theorem showing that under suitable conditions discrete-time random walk approximations to continuous-time optimal stopping problems can approximate 
the value function with an error of the order $O(\delta)$ and the stopping boundary with an error of the order $o(\sqrt{\delta})$, where $\delta$ is the interval width in discretizing time for the approximating random walk. The theorem also shows how to correct the discrete-time approximations to continuous-time stopping boundaries. As shown by Hogan [32] in a prototypical special case, the boundary correction involves an 'excess over the boundary' term. In this connection, we use renewal theory to derive in, Lemma 3.1, an inequality, which plays an important role in the proof of the theorem, for the excess over the boundary.

Suppose that in the continuous-time optimal stopping problem (2.1) with horizon $T^{*}$, we have an approximation $v_{\delta}(\cdot, T)$ to the value function $v(\cdot, T)$ and an approximation $\beta_{\delta}$ to $b(T)$, with $\widetilde{T}<t<T<T^{*}$, such that

$$
\beta_{\delta}=b(T)+O(\sqrt{\delta}), \quad v_{\delta}(x, T)-v(x, T)=O\left(\delta \mathrm{e}^{\alpha^{\prime}(x-b(T))^{-}}\right),
$$

uniformly in $x$ for some positive constant $\alpha^{\prime}$, where $x^{-}=\max \{-x, 0\}$. (Note that as $b(T)$ is approximated by $\beta_{\delta}$, we have $v_{\delta}(x, T)=g(x, T)$ for $x \geq \beta_{\delta}$, so $v_{\delta}(x, T)=v(x, T)=g(x, T)$ for $x \geq \max \left\{b(T), \beta_{\delta}\right\}$.) A random walk approximation to the value function and the stopping boundary of (2.1) for $\widetilde{T} \leq t \leq T$ can be described as follows. Let $t_{0}=T>t_{1}>\cdots>t_{K_{\delta}}=\widetilde{T}$ partition the interval into $K_{\delta}$ subintervals such that

$$
t_{i-1}-t_{i}=\delta \quad \text { for } 1 \leq i<K_{\delta}, t_{K_{\delta}-1}-t_{K_{\delta}} \leq \delta .
$$

Let $X, X_{1}, X_{2}, \ldots$ be independent and identically distributed (i.i.d.) random variables with

$$
\mathrm{E} X=0=\mathrm{E} X^{3}, \quad \mathrm{E} X^{2}=1, \quad \mathrm{Ee}^{\alpha^{\alpha^{\prime \prime}|X|}}<\infty \quad \text { for some } \alpha^{\prime \prime}>0,
$$

and let $S_{n}=X_{1}+\cdots+X_{n}\left(S_{0}=0\right)$. Approximating the Brownian motion in the optimal stopping problem (2.1) by the random walk $S_{n}$, we can use backward induction to compute the value function $v_{\delta}\left(x, t_{i}\right)$ and stopping boundary $b_{\delta}\left(t_{i}\right)$ of the discrete-time optimal stopping problem. Specifically, first initialize by defining $b_{\delta}\left(t_{i}\right)=\beta_{\delta}$ for $0 \leq i<|\log \delta|$. Then define recursively

$$
v_{\delta}\left(x, t_{i}\right)= \begin{cases}g\left(x, t_{i}\right) & \text { if } x \geq b_{\delta}\left(t_{i-1}\right)+\sqrt{\delta}|\log \delta|, \\ \max \left[g\left(x, t_{i}\right), \mathrm{E} v_{\delta}\left(x+\sqrt{\delta} X, t_{i-1}\right)\right] & \text { if }\left|x-b_{\delta}\left(t_{i-1}\right)\right|<\sqrt{\delta}|\log \delta|, \\ \mathrm{E} v_{\delta}\left(x+\sqrt{\delta} X, t_{i-1}\right) & \text { if } x \leq b_{\delta}\left(t_{i-1}\right)-\sqrt{\delta}|\log \delta|,\end{cases}
$$

for $1 \leq i \leq K_{\delta}$, and define $b_{\delta}\left(t_{i}\right)$ for $|\log \delta| \leq i \leq K_{\delta}$ recursively by

$$
\begin{aligned}
& b_{\delta}\left(t_{i}\right)=\inf \left\{x:\left|x-b_{\delta}\left(t_{i-1}\right)\right|<\sqrt{\delta}|\log \delta|,\right. \\
&\left.g\left(x, t_{i}\right) \geq \mathrm{E} v_{\delta}\left(x+\sqrt{\delta} X, t_{i-1}\right)\right\} \quad\left(\inf \varnothing=b_{\delta}\left(t_{i-1}\right)\right) .
\end{aligned}
$$

Remark 3.1. The assumption (2.2) on the continuous-time optimal stopping boundary $b(t)$ does not imply the existence of a corresponding discrete-time optimal stopping boundary below which is the continuation region. For this technical reason, (3.3) is used instead of the simpler (standard) backward induction algorithm $v_{\delta}\left(x, t_{i}\right)=\max \left[g\left(x, t_{i}\right), \mathrm{E} v_{\delta}\left(x+\sqrt{\delta} X, t_{i-1}\right)\right]$. (Thus, the resulting $v_{\delta}$ is not necessarily the value function of the discrete-time optimal stopping problem.) However, for specific applications (e.g. American put options and call options with $d>0$ ), such discrete-time optimal stopping boundaries can be shown to exist so that Theorem 3.1, below, still applies when (3.3) is replaced by the simpler backward induction algorithm. 
Theorem 3.1. With the same notation as in Section 2, assume that (2.2)-(2.5) hold, that $g_{*}(x) \geq$ 0 for all $x$, and that

$$
\begin{gathered}
G(b(t), t)<0 \quad \text { for all } \tilde{T} \leq t \leq T, \\
\sup _{x<b\left(T^{*}\right)} \mathrm{e}^{-\alpha^{\prime \prime \prime}\left|x-b\left(T^{*}\right)\right|}\left|g\left(x, T^{*}\right)\right|<\infty \quad \text { for some } \alpha^{\prime \prime \prime}>0, \\
\sup _{\tilde{T} \leq t \leq T^{*}, x \geq b(t)} \mathrm{e}^{-\beta(x)}\{|g(x, t)|+|G(x, t)|\}<\infty,
\end{gathered}
$$

with $\beta(x)=O(x)$ as $x \rightarrow \infty$. Then

$$
\begin{gathered}
\sup _{1 \leq i \leq K_{\delta}, x \in \mathbb{R}} \mathrm{e}^{-\alpha\left(x-b\left(t_{i}\right)\right)^{-}}\left|v_{\delta}\left(x, t_{i}\right)-v\left(x, t_{i}\right)\right|=O(\delta) \quad \text { with } \alpha:=\max \left\{\alpha^{\prime}, \alpha^{\prime \prime \prime}\right\}, \\
b_{\delta}\left(t_{i}\right)=b\left(t_{i}\right)-\rho \sqrt{\delta}+o(\sqrt{\delta}) \quad \text { uniformly in }|\log \delta| \leq i \leq K_{\delta},
\end{gathered}
$$

where $\tau_{+}=\inf \left\{n: S_{n}>0\right\}$ and $\rho=\mathrm{E} S_{\tau_{+}}^{2} / 2 \mathrm{E} S_{\tau_{+}}$.

Remark 3.2. In order to apply (2.10), we need the condition that $g^{*}(x) \geq 0$ for all $x$, which is satisfied in typical applications; see [49, p. 105]. In view of Lemma 2.1(a), $G(b(t), t) \leq 0$ and condition (3.5) requires the inequality to be strict for all $t \in[\widetilde{T}, T]$. Conditions (3.6) and (3.7) are assumed in order to establish bounds on higher-order derivatives of $v$ with respect to $x$; see Lemma 3.7. By (3.8), we have $v_{\delta}\left(x, t_{i}\right)-v\left(x, t_{i}\right)=O(\delta)$ uniformly in $i \in\left\{1, \ldots, K_{\delta}\right\}$ and in $x$ over any bounded subset of $\mathbb{R}$. Along the lines of the proof of Theorem 3.1, it can be shown that $\sup _{1<i<K_{\delta}, x \in \mathbb{R}}\left|v_{\delta}\left(x, t_{i}\right)-v\left(x, t_{i}\right)\right|=O(\delta)$, if (3.1) and (3.6) are strengthened to $\sup _{x}\left|v_{\delta}(x, T)-v(x, T)\right|=O(\delta)$ and $\sup _{x<b\left(T^{*}\right)}\left|g\left(x, T^{*}\right)\right|<\infty$. The latter condition implies that $v(x, t)$ has a bounded fifth derivative with respect to $x$ in the continuation region; see Lemma 3.7.

Remark 3.3. In many applications, the payoff function may not belong to the class $C^{3,2}$ in the continuation region. For example, the payoff function associated with the standard American call option is given by $g(x, t)=\mathrm{e}^{-r t}\left(P_{0} \mathrm{e}^{\left(r-d-\sigma^{2} / 2\right) t+\sigma x}-K\right)^{+}$, which is not differentiable in $\left\{(x, t): P_{0} \mathrm{e}^{\left(r-d-\sigma^{2} / 2\right) t+\sigma x}=K\right\}$. It can be readily shown that the value function and the stopping boundary remain unchanged if the payoff function is replaced in the continuation region by any function not greater than the value function (cf. [49, p. 143]). Therefore, this payoff function yields the same value function and stopping boundary as $\tilde{g}(x, t)=$ $\mathrm{e}^{-r t}\left(P_{0} \mathrm{e}^{\left(r-d-\sigma^{2} / 2\right) t+\sigma x}-K\right) \mathbf{1}_{\left\{t<T^{*}\right\}}+g\left(x, T^{*}\right) \mathbf{1}_{\left\{t=T^{*}\right\}}$, to which Theorem 3.1 applies. Moreover, such changes in the payoff function (in the continuation region) have little effect on the algorithm (3.3) and (3.4) if $\delta$ is sufficiently small. Furthermore, as noted in Remark 3.1, for American call (and put) options, Theorem 3.1 still applies when (3.3) is replaced by the standard backward induction algorithm. Then the discrete-time value function with respect to the payoff function $g$ is the same as that with respect to $\tilde{g}$ as long as the initialization $v_{\delta}(x, T)$ is nonnegative for all $x$. To see this for an American call option with $d>0$, note that it is not optimal for the random walk to stop in the region with $P_{t}<K$ and $t<T$. This comment also applies to an American put option.

Remark 3.4. Although $v_{\delta}(x, t)$ only gives an approximation to $v(x, t)$ for $t=t_{i}, i=0, \ldots$, $K_{\delta}$, we can extend $v_{\delta}(x, t)$ by linear interpolation to approximate $v(x, t)$ for $t_{i}<t<t_{i-1}$, where we define

$$
v_{\delta}(x, t)=\frac{t_{i-1}-t}{t_{i-1}-t_{i}} v_{\delta}\left(x, t_{i}\right)+\frac{t-t_{i}}{t_{i-1}-t_{i}} v_{\delta}\left(x, t_{i-1}\right) .
$$


With this definition of $v_{\delta}(x, t)$ at every $t \in[\widetilde{T}, T]$, the $O(\delta)$ error in (3.8) still holds if we replace $v_{\delta}\left(x, t_{i}\right)-v\left(x, t_{i}\right)$ by $v_{\delta}(x, t)-v(x, t)$ and take the supremum over $t \in[\widetilde{T}, T]$. In practice, $X$ is usually taken to be lattice-valued to avoid numerical integration in computing $\mathrm{E} v_{\delta}\left(x+\sqrt{\delta} X, t_{i-1}\right)$. In this case, with $L$ denoting the lattice for which $\mathrm{P}(X \in L)=1$, $v_{\delta}(x, t)$ is only given by (3.3) for $x \in \sqrt{\delta} L$ if $(x, t)$ belongs to the continuation region of the discrete-time optimal stopping problem. For $x_{j}<x<x_{j+1}$, where $x_{j}$ and $x_{j+1}$ are two adjacent points of the lattice $\sqrt{\delta} L$ such that at least one of $\left(x_{j}, t\right)$ and $\left(x_{j+1}, t\right)$ is a continuation point, we can again use linear interpolation to approximate $v(x, t)$ by

$$
\widehat{v}_{\delta}(x, t)=\frac{x_{j+1}-x}{x_{j+1}-x_{j}} v_{\delta}\left(x_{j}, t\right)+\frac{x-x_{j}}{x_{j+1}-x_{j}} v_{\delta}\left(x_{j+1}, t\right) ;
$$

see Section 3.4 for further details and discussion.

\subsection{Overshoots, ladder heights, and a renewal-theoretic lemma}

In the nonarithmetic case, as noted by Hogan [32], the constant $\rho$ in Theorem 3.1 is the limiting expected overshoot of the boundary $c$ at the first passage time $\tau(c)=\inf \left\{n: S_{n}>c\right\}$. Specifically $\rho=\lim _{c \rightarrow \infty}$ E $R_{c}$ in this case, where $R_{c}=S_{\tau(c)}-c$. Moreover, in the case where $X$ is arithmetic with span $h$, E $R_{c} \rightarrow \rho+h / 2$ as $c \rightarrow \infty$ in $L$; see [24, p. 300, 371]. Whereas only $\lim _{c \rightarrow \infty} \mathrm{E} R_{c}$ is involved in approximating the difference between $b_{\delta}(t)$ and $b(t)$, bounds on $\mathrm{E} R_{c}$ for all values of $c$ are needed in the proof of Theorem 3.1. Lorden [41] proved the upper bound $\sup _{c \geq 0} \mathrm{E} R_{c} \leq \mathrm{E} S_{\tau_{+}}^{2} / \mathrm{E} S_{\tau_{+}}(=2 \rho)$ by making use of Wald's identity and certain representations of $\int_{0}^{c} R_{t} \mathrm{~d} t$. He also obtained similar inequalities for $\sup _{c \geq 0} \mathrm{E} R_{c}^{p}$ for any $p>0$; in particular

$$
\sup _{c \geq 0} R_{c}^{2} \leq \frac{4 \mathrm{E} S_{\tau_{+}}^{3}}{3 \mathrm{E} S_{\tau_{+}}}
$$

which is also used in the proof of Theorem 3.1. Note that $\mathrm{E} S_{\tau_{+}}^{p}<\infty$ if E $X=0$ and $\mathrm{E}\left(X^{+}\right)^{p+1}<\infty$; see [16, Theorem 1]. Besides (3.10), we also need a different inequality which bounds $\mathrm{E} R_{c}^{2}$ by $\rho$ and $\mathrm{E} R_{c}$ and which is used in the proof of Lemma 3.5.

Lemma 3.1. If $\mathrm{E} X>0$ and $\mathrm{E}\left(X^{+}\right)^{2}<\infty$, or if $\mathrm{E} X=0$ and $0<\mathrm{E}\left(X^{+}\right)^{3}<\infty$, then $\mathrm{E} R_{c}^{2} \leq 2 \rho\left(c+\mathrm{E} R_{c}\right)$ for all $c \geq 0$.

Proof. Under the assumptions on $X, \tau(c)<\infty$ almost surely and $\mathrm{E} S_{\tau(c)}^{2}<\infty$ (cf. [30, Theorem 2.1], for the case in which $\mathrm{E} X>0$ and [16, Theorem 1], for the case in which E $X=0)$. Note that $\tau_{+}=\tau(0)$. Replacing $X$ by the ladder variable $S_{\tau_{+}}$, we shall assume, without loss of generality, that $X>0$ and $\mathrm{E} X^{2}<\infty$. For the positive random variable $X$, the desired inequality $\mathrm{E} R_{c}^{2} \leq 2 \rho\left(c+\mathrm{E} R_{c}\right)$ can be written as

$$
(\mathrm{E} X)\left(\mathrm{E} R_{c}^{2}\right) \leq\left(\mathrm{E} X^{2}\right)\left(c+\mathrm{E} R_{c}\right)
$$

which we shall prove by using the renewal function $U(x)=\sum_{n=0}^{\infty} \mathrm{P}\left(S_{n} \leq x\right), S_{0}=0$. Let $F$ denote the distribution function of $X$ and let

$$
G(t)=\int_{t}^{\infty} x(1-F(x)) \mathrm{d} x, \quad H(t)=\int_{t}^{\infty}(1-F(x)) \mathrm{d} x, \quad t \geq 0 .
$$

To prove that (3.11) holds, note that, for $y \geq 0$,

$$
\mathrm{P}\left(R_{c}>y\right)=\sum_{n=1}^{\infty} \mathrm{P}\left(S_{n-1} \leq c, S_{n}>c+y\right)=\int_{[0, c]}\{1-F(y+c-z)\} \mathrm{d} U(z),
$$


in which $U$ is interpreted as a measure with $U(\{0\})=1$, and therefore,

$$
\begin{aligned}
\mathrm{E} R_{c}^{2} & =2 \int_{0}^{\infty} y \int_{[0, c]}\{1-F(y+c-z)\} \mathrm{d} U(z) \mathrm{d} y \\
& =2 \int_{[0, c]} \int_{c-z}^{\infty}(x-c+z)(1-F(x)) \mathrm{d} x \mathrm{~d} U(z) \\
& =2 \int_{[0, c]} G(c-z) \mathrm{d} U(z)-2 \int_{[0, c]}(c-z) H(c-z) \mathrm{d} U(z), \\
\mathrm{E} R_{c} & =\int_{0}^{\infty} \int_{[0, c]}\{1-F(y+c-z)\} \mathrm{d} U(z) \mathrm{d} y \\
& =\int_{[0, c]} H(c-z) \mathrm{d} U(z) .
\end{aligned}
$$

Since $\int_{[0, c]} G(c-z) \mathrm{d} U(z)=G(c)+\int_{(0, c]} G(c-z) \mathrm{d} U(z)$ and since $U(0)=1, G(0)=\frac{1}{2} \mathrm{E} X^{2}$ and $(\mathrm{d} / \mathrm{d} z) G(c-z)=(c-z)(1-F(c-z))$ almost everywhere, integration by parts yields

$$
\begin{aligned}
\mathrm{E} R_{c}^{2}= & \left(\mathrm{E} X^{2}\right) U(c)-2 \int_{[0, c]} U(z)(c-z)(1-F(c-z)) \mathrm{d} z \\
& -2 \int_{[0, c]}(c-z) H(c-z) \mathrm{d} U(z) \\
\leq & \left(\mathrm{E} X^{2}\right) U(c)
\end{aligned}
$$

Since $H(c-z)=\mathrm{E} X-\int_{0}^{c-z}(1-F(x)) \mathrm{d} x=\mathrm{E} X-\int_{z}^{c}(1-F(t-z)) \mathrm{d} t$ for $0 \leq z \leq c$,

$$
\begin{aligned}
\mathrm{E} R_{c} & =(\mathrm{E} X) \int_{[0, c]} \mathrm{d} U(z)-\int_{[0, c]} \int_{z}^{c}(1-F(t-z)) \mathrm{d} t \mathrm{~d} U(z) \\
& =(\mathrm{E} X) U(c)-\int_{0}^{c} \int_{[0, t]}(1-F(t-z)) \mathrm{d} U(z) \mathrm{d} t .
\end{aligned}
$$

Noting that $\int_{[0, t]}(1-F(t-z)) \mathrm{d} U(z)=U(t)-\int_{[0, t]} F(t-z) \mathrm{d} U(z)$ and $\int_{[0, t]} F(t-z) \mathrm{d} U(z)=$ $\sum_{n=1}^{\infty} \mathrm{P}\left(S_{n} \leq t\right)=U(t)-1$, we then obtain

$$
c+\mathrm{E} R_{c}=c+(\mathrm{E} X) U(c)-\int_{0}^{c} \mathrm{~d} t=(\mathrm{E} X) U(c) .
$$

From (3.12) and (3.13), the desired conclusion (3.11) follows.

\subsection{A canonical optimal stopping problem}

We introduce here a canonical optimal stopping problem, depending on $\delta$ and $0<\zeta<\gamma<$ $\frac{1}{3}$, which is basic to the induction proof of Theorem 3.1. The payoff function is of the form

$$
g_{\delta}(y, s)=\left[-s+r_{\delta}(y, s)\right] \mathbf{1}_{\{s<0\}}+h_{\delta}(y) \mathbf{1}_{\{s=0\}},
$$

where, for some $A>0$,

$$
\begin{gathered}
\left|r_{\delta}(y, s)\right| \leq A\left\{\delta s^{2}+\sqrt{\delta}|s y|+\sqrt{\delta}|y|^{3}\right\} \quad \text { for }|y| \leq \delta^{-1 / 6}, \\
h_{\delta}(y)=y^{2} \mathbf{1}_{\{y \leq 0\}}+a_{\delta}(y) \quad \text { with }\left|a_{\delta}(y)\right| \leq A \quad \text { for }|y| \leq \delta^{-1 / 6} .
\end{gathered}
$$


Furthermore assume that, for the random walk $\left\{S_{n}, n \geq 1\right\}$,

$$
\sup _{|y| \leq \delta^{-\zeta / 2}, n \leq \delta^{-\gamma}} \mathrm{E}\left|h_{\delta}\left(y+S_{n}\right)\right|^{p}+\sup _{|y| \leq \delta^{-\zeta / 2}, 1 \leq m \leq \delta^{-\gamma}, 1 \leq n \leq \delta^{-\gamma}} \mathrm{E}\left|r_{\delta}\left(y+S_{n},-m\right)\right|^{p}=O\left(\delta^{-q}\right)
$$

for some $p>1$ and $q>0$. For $-\infty<y<\infty$ and $t \in\{0,-1,-2, \ldots\}$, let

$$
V_{\delta}(y, t)=\sup _{\tau \in \mathcal{T}_{\delta, t, y}} \mathrm{E} g_{\delta}\left(y+S_{\tau}, t+\tau\right)
$$

where $\mathcal{T}_{\delta, t, y}$ is the class of integer-valued stopping times $\tau \leq-t$ such that, on $\{-t>\tau \geq n\}$,

$$
\begin{aligned}
y+S_{n} \geq|\log \delta| & \Rightarrow \text { stopping occurs at } n, \\
y+S_{n}<-|\log \delta| & \Rightarrow \text { stopping cannot occur at } n .
\end{aligned}
$$

This canonical optimal stopping problem is a modification of Chernoff's [11] associated stopping problem with payoff function $g$ defined in (1.1), which is a special case of (3.14). Note that

$$
\widetilde{V}(y, t):=\sup _{0 \leq \tau \leq-t} \mathrm{E} g\left(y+S_{\tau}, t+\tau\right)+t=\sup _{0 \leq \tau \leq-t} \mathrm{E}\left[g^{*}\left(y+S_{\tau}\right) \mathbf{1}_{\{t+\tau=0\}}-\tau\right],
$$

where $g^{*}(y)=y^{2} \mathbf{1}_{\{y \leq 0\}}$, and that the associated optimal stopping problem with payoff function (1.1) has value function $\widetilde{V}(y, t)-t$.

As pointed out by Hogan [32], Lemmas 3.1 and 3.2 of [11] hold for general zero-mean, finitevariance random walks. Therefore, $\widetilde{V}(y, t)$ is monotone decreasing in $-t$ and in $y$. Hogan [32] has shown that there exists a nondecreasing optimal stopping boundary $z(t)<0$ for $t<0$ such that $\lim _{t \rightarrow-\infty} z(t)=-\rho, \widetilde{V}(y, t)>0$ if $y<z(t)$, and $\widetilde{V}(y, t)=0$ if $y \geq z(t)$. The following lemma, whose proof is given in Appendix A, considers instead of $\widetilde{V}(y, t)$ the expected reward $u(y, t)$ of the policy that starts at $(y, t)$, takes an observation, and proceeds optimally thereafter.

Lemma 3.2. For $t<0$, let $u(y, t)=-1+\mathrm{E} \widetilde{V}(y+X, t+1)$. Then $u(y, t)<0$ for $y>z(t)$. Consequently, $\lim _{t \rightarrow-\infty} u(y, t)<0$ for every $y>-\rho$.

To analyze the canonical optimal stopping problem (3.18), we use Lemmas 3.1, 3.2, and the following result.

Lemma 3.3. Let $\tau(y)=\inf \left\{n: S_{n}>y\right\}$, in which $S_{n}=\sum_{i=1}^{n} X_{i}$ and $X, X_{1}, \ldots$ are i.i.d. random variables satisfying (3.2). Then, as $m \rightarrow \infty$,

(i) $\mathrm{P}(\tau(y) \geq m)=O\left(\left(y^{2} / m\right)^{\lambda}\right)$ uniformly in $1 \leq y \leq \sqrt{m}$ for every $\lambda<\frac{1}{2}$,

(ii) $\mathrm{P}(\tau(y) \leq m) \leq \exp \left\{-\varepsilon \min \left(y^{2} / m, \varepsilon y / 2\right)\right\}$ for some $\varepsilon>0$ and all $y \geq \sqrt{m}$.

Lemma 3.3(i) follows from [37, Equations (4.6), (4.12), and (4.13)]. Lemma 3.3(ii) follows from $\mathrm{P}(\tau(y) \leq m)=\mathrm{P}\left(\max _{n \leq m} S_{n}>y\right) \leq \mathrm{e}^{-\theta y}\left(\mathrm{E} \mathrm{e}^{\theta X}\right)^{m}$, by the submartingale inequality and (3.2), with $\theta>0$ and $\varepsilon>0$ chosen suitably to yield the desired conclusion. In Appendix A we make use of Lemmas 3.1-3.3 to prove the following results on the canonical optimal stopping problem (3.18) that are used to prove Theorem 3.1.

Lemma 3.4. For every fixed $\varepsilon>0$ and all sufficiently small $\delta>0$, if $-\delta^{-\gamma} \leq t \leq-|\log \delta|$ then, in the optimal stopping problem (3.18), $(y, t)$ is a stopping point for $y>-\rho+\varepsilon$.

Lemma 3.5. For every fixed $\varepsilon>0$ and all sufficiently small $\delta>0$, if $-\delta^{-\gamma} \leq t \leq-|\log \delta|$ then, in the optimal stopping problem (3.18), $(y, t)$ is a continuation point for $y<-\rho-\varepsilon$. 


\subsection{Proof of Theorem 3.1}

To prove Theorem 3.1 we approximate the underlying optimal stopping problem by a recursively defined family of optimal stopping problems. The payoff function in each optimal stopping problem is of the canonical form (3.14), in which $g_{\delta}$ is given by the following result.

Lemma 3.6. With the same notation and assumptions as in Theorem 3.1, let $t^{*} \in(\widetilde{T}, T]$ and $x^{*}=b\left(t^{*}\right)$. Define the quadratic function

$\pi_{g}(x, t)=g\left(x^{*}, t^{*}\right)+\frac{\partial g}{\partial x}\left(x^{*}, t^{*}\right)\left(x-x^{*}\right)+\frac{1}{2} \frac{\partial^{2} g}{\partial x^{2}}\left(x^{*}, t^{*}\right)\left(x-x^{*}\right)^{2}-\frac{1}{2} \frac{\partial^{2} g}{\partial x^{2}}\left(x^{*}, t^{*}\right)\left(t-t^{*}\right)$.

Letting $y=\delta^{-1 / 2}\left(x-x^{*}\right)$ and $s=\delta^{-1}\left(t-t^{*}\right)$, define

$$
\tilde{g}_{\delta}(y, s)=\delta^{-1}\left\{g(x, t)-\pi_{g}(x, t)\right\} \mathbf{1}_{\left\{t<t^{*}\right\}}+\left[\delta^{-1}\left\{v(x, t)-\pi_{g}(x, t)\right\}+C_{\delta}(y)\right] \mathbf{1}_{\left\{t=t^{*}\right\}},
$$

where $\exp \left\{-\alpha \sqrt{\delta} y^{-}\right\}\left|C_{\delta}(y)\right| /\left|G\left(x^{*}, t^{*}\right)\right|$ is bounded by some positive constant $A$ for all $y$ and all sufficiently small $\delta>0$ with $\alpha$ given in (3.8). Then $g_{\delta}:=\widetilde{g}_{\delta} /\left|G\left(x^{*}, t^{*}\right)\right|$ satisfies (3.14)(3.17) for $-\delta^{-\gamma} \leq s \leq 0$, for every choice of $0<\zeta<\gamma<\frac{1}{3}$, and $q \geq p>1$. Furthermore, defining $V_{\delta}$ by (3.18) with this choice of $g_{\delta}$, we have, as $\delta \rightarrow 0$,

(i) $V_{\delta}(y, s)=y^{2}-s-\mathrm{E}\left(-\rho+R_{-\rho-y}\right)^{2}+o(1)$ uniformly in $-\delta^{-\gamma} \leq s \leq-\frac{1}{2} \delta^{-\gamma}$ and $-\rho \geq y \geq-\delta^{-\zeta / 2}$

(ii) $V_{\delta}(y, s)=y^{2} \mathbf{1}_{\{y \leq 0\}}-s+O$ (1) uniformly in $-\delta^{-\gamma} \leq s<0$ and $|y| \leq \delta^{-\zeta / 2}$.

Remark 3.5. For the symmetric Bernoulli case, the term $\mathrm{E}\left(-\rho+R_{-\rho-y}\right)^{2}$ appearing in Lemma 3.6(i) plays an important role in Chernoff and Petkau's numerical algorithm [15, p. 48] for computing the boundary $b(\cdot)$.

Remark 3.6. Letting $y=\delta^{-1 / 2}\left(x-x^{*}\right)$ and $s=\delta^{-1}\left(t-t^{*}\right)$, define $V(y, s)=\delta^{-1}(v(x, t)-$ $\left.\pi_{g}(x, t)\right) /\left|G\left(x^{*}, t^{*}\right)\right|$. Recalling that $y^{2} \mathbf{1}_{\{y \leq 0\}}-s$ is the (continuous-time) value function for the optimal stopping problem (1.1), we can make use of Taylor expansions and arguments as in the proof of Lemma 3.6 (given in Appendix A) to show that $V(y, s)=y^{2} \mathbf{1}_{\{y \leq 0\}}-s+o(1)$ uniformly in $-\delta^{-\gamma} \leq s<0$ and $|y| \leq \delta^{-\zeta / 2}$. By Lemma 3.6(ii), there exist $C>0$ and $\delta_{0}>0$ (depending on the constant $A$ that bounds $\exp \left\{-\alpha \sqrt{\delta} y^{-}\right\}\left|C_{\delta}(y)\right| /\left|G\left(x^{*}, t^{*}\right)\right|$ ) such that, for $0<\delta<\delta_{0}$, we have $\left|V(y, s)-V_{\delta}(y, s)\right| \leq C$ for $-\delta^{-\gamma} \leq s<0$ and $|y| \leq \delta^{-\zeta / 2}$. Moreover, by Lemma 3.6(i), there exists $\delta_{1}>0$ (depending on $A$ ) such that for $0<\delta<\delta_{1}$, we have

$$
\left|V(y, s)-V_{\delta}(y, s)\right| \leq 1+\sup _{y<-\rho} \mathrm{E}\left(-\rho+R_{-\rho-y}\right)^{2},
$$

for $-\delta^{-\gamma} \leq s \leq-\frac{1}{2} \delta^{-\gamma}$ and $-\rho \geq y \geq-\delta^{-\zeta / 2}$. Since, by Lemma 3.4, $V_{\delta}(y, s)=-s+o(1)$ for $-\rho<y \leq \delta^{-\zeta / 2}$ and since $\rho^{2}=\mathrm{E}\left(-\rho+R_{0}\right)^{2}=\mathrm{E}\left(-\rho+S_{\tau_{+}}\right)^{2}$, the inequality (3.21) actually holds for $-\delta^{-\gamma} \leq s \leq-\frac{1}{2} \delta^{-\gamma}$ and $|y| \leq \delta^{-\zeta / 2}$.

We next combine Lemma 2.2 with conditions (3.6) and (3.7) of Theorem 3.1 to prove the following result.

Lemma 3.7. With the same notation and assumptions as in Theorem 3.1,

$$
\sup _{\widetilde{T} \leq t \leq T, x<b(t)} \mathrm{e}^{-\alpha^{\prime \prime \prime}|x-b(t)|}\left|\frac{\partial^{j}}{\partial x^{j}} v(x, t)\right|<\infty \text { for } j=0, \ldots, 5,
$$


where $\left(\partial^{0} / \partial x^{0}\right) v(x, t):=v(x, t)$. Furthermore, if (3.6) is strengthened to

$$
\sup _{x<b\left(T^{*}\right)}\left|g\left(x, T^{*}\right)\right|<\infty
$$

then we have

$$
\sup _{\widetilde{T} \leq t \leq T, x<b(t)}\left|\frac{\partial^{j}}{\partial x^{j}} v(x, t)\right|<\infty \text { for } j=0, \ldots, 5 .
$$

Proof. By Lemma 2.2, $\sup _{\widetilde{T} \leq t \leq T, L \leq x<b(t)}\left|\left(\partial^{j} / \partial x^{j}\right) v(x, t)\right|<\infty$ for $j=0, \ldots, 5$, where $L:=\min \{b(t): \widetilde{T} \leq t \leq T\}-1$. Let $\Omega=\{(x, t): x \leq L, \widetilde{T} \leq t \leq T\}$. Note that $\mathrm{E}_{x, t} g\left(B_{T^{*}}, T^{*}\right)$ can be expressed as

$$
\int_{-\infty}^{b\left(T^{*}\right)} g\left(u, T^{*}\right) \phi\left(\frac{u-x}{\sqrt{T^{*}-t}}\right) \frac{\mathrm{d} u}{\sqrt{T^{*}-t}}+\int_{b\left(T^{*}\right)}^{\infty} g\left(u, T^{*}\right) \phi\left(\frac{u-x}{\sqrt{T^{*}-t}}\right) \frac{\mathrm{d} u}{\sqrt{T^{*}-t}} .
$$

Here, in view of (3.7) and $T<T^{*}$, the second integral has a bounded $j$ th derivative with respect to $x$ over $\Omega(j=0, \ldots, 5)$, whereas the first integral has its $j$ th derivative bounded by $O\left(\mathrm{e}^{\alpha^{\prime \prime \prime}|x|}\right)$ over $\Omega$ in view of (3.6) (or bounded by $O(1)$ if $\sup _{x<b\left(T^{*}\right)}\left|g\left(x, T^{*}\right)\right|<\infty$ ). Similarly,

$$
\int_{t}^{T^{*}}\left\{\int_{b(s)}^{\infty} G(u, s) \phi\left(\frac{u-x}{\sqrt{s-t}}\right) \frac{\mathrm{d} u}{\sqrt{s-t}}\right\} \mathrm{d} s
$$

also has a bounded $j$ th derivative with respect to $x$ over $\Omega, j=0, \ldots, 5$. Hence, the desired conclusion follows from (2.10).

Proof of Theorem 3.1. Let $\varepsilon>0$ and take $0<\zeta<\gamma<\frac{1}{3}$. We shall use induction to prove that, for sufficiently small $\delta>0$,

$$
\begin{aligned}
& \left|b\left(t_{i}\right)-b_{\delta}\left(t_{i}\right)-\rho \sqrt{\delta}\right| \leq \varepsilon \sqrt{\delta}, \quad i=[|\log \delta|], \ldots, K_{\delta}, \\
& \max _{b\left(t_{i-1}\right)-\delta^{(1-\zeta) / 2} \leq x \leq b\left(t_{i-1}\right)+2 \delta^{1 / 2}|\log \delta|}\left|v\left(x, t_{i}\right)-v_{\delta}\left(x, t_{i}\right)\right| \leq C_{0} \delta, \quad i=1, \ldots, K_{\delta}, \\
& \max _{x<b\left(t_{i-1}\right)-\delta^{(1-\zeta) / 2}} \mathrm{e}^{-\alpha\left|x-b\left(t_{i}\right)\right|}\left|v\left(x, t_{i}\right)-v_{\delta}\left(x, t_{i}\right)\right| \leq \mathrm{e}^{\alpha B \delta i}\left(C_{0} \delta+C_{1} \delta^{2} i\right), \quad i=1, \ldots, K_{\delta},
\end{aligned}
$$

where $B:=\max \left\{\left|b^{\prime}(t)\right|: \widetilde{T} \leq t \leq T\right\}<\infty$ and $C_{0}$ and $C_{1}$ are positive constants that will be specified later. Since $\left\{\pi_{g}\left(x+\sqrt{\delta} S_{n}, t+n \delta\right), n \geq 0\right\}$ and $\left\{\pi_{g}\left(x+\sqrt{\delta} B_{u}, t+\delta u\right), u \geq 0\right\}$ are martingales, Doob's optional stopping theorem yields $\pi_{g}(x, t)=\mathrm{E} \pi_{g}\left(x+\sqrt{\delta} B_{\tau}, t+\delta \tau\right)=$ $\mathrm{E} \pi_{g}\left(x+\sqrt{\delta} S_{\kappa}, t+\delta \kappa\right)$ for any bounded stopping times $\kappa$ (adapted to the filtration generated by $\left.\left\{S_{n}\right\}\right)$ and $\tau$. In the induction proof of (3.22)-(3.24), we can therefore replace $g$ and $v$ by $g-\pi_{g}$ and $v-\pi_{g}$ for each time interval $\left[t_{i}, t_{i-1}\right]$ with the optimal stopping boundary preserved. Note also that by (2.4), (3.3), and (3.22), we have, for $i=1, \ldots, K_{\delta}$,

$$
b\left(t_{i-1}\right)+2 \delta^{1 / 2}|\log \delta|>\max \left\{b\left(t_{i}\right), b_{\delta}\left(t_{i-1}\right)+\delta^{1 / 2}|\log \delta|\right\},
$$

for sufficiently small $\delta>0$, from which it follows that $v\left(x, t_{i}\right)=v_{\delta}\left(x, t_{i}\right)=g\left(x, t_{i}\right)$, for $x>b\left(t_{i-1}\right)+2 \delta^{1 / 2}|\log \delta|$. 
We first apply Lemma 3.6 with $t^{*}=T\left(=t_{0}\right), y=\delta^{-1 / 2}(x-b(T))$, and $C_{\delta}(y)=$ $\delta^{-1}\left\{v_{\delta}(x, T)-v(x, T)\right\}$. By (3.1) and noting that $\alpha^{\prime} \leq \alpha$, we have

$$
A:=1+\limsup _{\delta \rightarrow 0} \sup _{y} \frac{\exp \left\{-\alpha \sqrt{\delta} y^{-}\right\}\left|C_{\delta}(y)\right|}{|G(b(T), T)|}<\infty .
$$

Then Lemmas 3.4, 3.5, and 3.6(ii) show that (3.22) holds for $i=[\log \delta], \ldots,\left[\delta^{-\gamma}\right]$ and that (3.23) holds for $i=1, \ldots,\left[\delta^{-\gamma}\right]$ for some constant $C_{0}$ (see Remark 3.6). We shall choose $C_{0}$ to satisfy

$$
C_{0} \geq \sup _{\widetilde{T} \leq t \leq T}|G(b(t), t)| \max \left\{A, 1+\sup _{y<-\rho} \mathrm{E}\left(-\rho+R_{-\rho-y}\right)^{2}\right\} .
$$

We now make use of (3.23) and an induction argument to prove (3.24) for $i=1, \ldots,\left[\delta^{-\gamma}\right]$. First consider the case in which $i=1$. For $x \leq b(T)-\delta^{(1-\zeta) / 2}, v_{\delta}\left(x, t_{1}\right)=\mathrm{E} v_{\delta}(x+\sqrt{\delta} X, T)$ (by (3.3)), and $v\left(x, t_{1}\right)=\mathrm{E} v\left(x+\sqrt{\delta} B_{1}, T\right)+O\left(\delta^{p}\right)$ for every $p>0$. It follows that, for $x \leq b(T)-\delta^{(1-\zeta) / 2}$,

$$
v_{\delta}\left(x, t_{1}\right)-v\left(x, t_{1}\right)=\mathrm{E}\left[v_{\delta}(x+\sqrt{\delta} X, T)-v\left(x+\sqrt{\delta} B_{1}, T\right)\right]+O\left(\delta^{4}\right) .
$$

By (3.25), $\sup _{x} \exp \left\{-\alpha(x-b(T))^{-}\right\}\left|v_{\delta}(x, T)-v(x, T)\right| \leq C_{0} \delta$ for sufficiently small $\delta>0$. Since $\mathrm{E} X^{r}=\mathrm{E} B_{1}^{r}$ for $r=1,2,3$ by (3.2), it follows, from Lemma 3.7 and Taylor's theorem, that

$$
\begin{aligned}
\mathrm{E}[v(x+ & \sqrt{\delta} X, T)-v(x, T)]-\mathrm{E}\left[v\left(x+\sqrt{\delta} B_{1}, T\right)-v(x, T)\right] \\
= & \frac{1}{4 !} \frac{\partial^{4} v}{\partial x^{4}}(x, T)\left\{\mathrm{E}(\sqrt{\delta} X)^{4}-\mathrm{E}\left(\sqrt{\delta} B_{1}\right)^{4}\right\} \\
& +\frac{1}{5 !} \mathrm{E}\left[\frac{\partial^{5} v}{\partial x^{5}}\left(x+\theta^{\prime} \sqrt{\delta} X, T\right)(\sqrt{\delta} X)^{5}-\frac{\partial^{5} v}{\partial x^{5}}\left(x+\theta^{\prime \prime} \sqrt{\delta} B_{1}, T\right)\left(\sqrt{\delta} B_{1}\right)^{5}\right],
\end{aligned}
$$

where $\theta^{\prime}$ and $\theta^{\prime \prime}$ are between 0 and 1. (Strictly speaking, as $\partial^{2} v(x, T) / \partial x^{2}$ has a discontinuity at the boundary, we need to replace $v(x, T)$ by a fifth polynomial of $x$ in the half line $\{(x, T): b(T) \leq x<\infty\}$ so as to make the fifth derivative continuous in order to apply Taylor's theorem. Such replacement yields a negligible error of $O\left(\delta^{p}\right)$ for every $p>0$.) Setting

$$
C_{1}=1+\frac{1}{4 !}\left|\mathrm{E} X^{4}-\mathrm{E} B_{1}^{4}\right| \sup _{\widetilde{T} \leq t \leq T, x<b(t)} \mathrm{e}^{-\alpha|x-b(t)|}\left|\frac{\partial^{4} v}{\partial x^{4}}(x, t)\right|,
$$

we have, by (3.26) and (3.27) together with Lemma 3.7,

$$
\sup _{x<b(T)-\delta^{(1-\zeta) / 2}} \mathrm{e}^{-\alpha|x-b(T)|}\left|v_{\delta}\left(x, t_{1}\right)-v\left(x, t_{1}\right)\right| \leq C_{0} \delta+C_{1} \delta^{2}
$$

for sufficiently small $\delta>0$, implying that

$$
\sup _{x<b(T)-\delta^{(1-\zeta) / 2}} \mathrm{e}^{-\alpha\left|x-b\left(t_{1}\right)\right|}\left|v_{\delta}\left(x, t_{1}\right)-v\left(x, t_{1}\right)\right| \leq \mathrm{e}^{\alpha B \delta}\left(C_{0} \delta+C_{1} \delta^{2}\right) .
$$

This shows that (3.24) holds for $i=1$. Replacing $T\left(=t_{0}\right)$ in the preceding argument by $t_{1}$ then shows that (3.24) holds for $i=2$, recalling that (3.23) has already been established for $i=1$. Proceeding inductively in this way proves that (3.24) holds for $i=1, \ldots,\left[\delta^{-\gamma}\right]$. 
To proceed inductively beyond $\left[\delta^{-\gamma}\right]$, suppose that (3.22)-(3.24) have been shown to hold for all $i \leq j$ with $j \geq\left[\delta^{-\gamma}\right]$. To show that (3.22) and (3.23) also hold for $i=j+1$, we apply Lemma 3.6 with $t^{*}=t_{j-J}$ and $C_{\delta}(y)=\delta^{-1}\left\{v_{\delta}\left(x, t_{j-J}\right)-v\left(x, t_{j-J}\right)\right\}$, where $J=\left[\delta^{-\gamma} / 2\right]$. By the induction hypothesis, we have

$$
\sup _{y} \mathrm{e}^{-\alpha \sqrt{\delta} y^{-}} \frac{\left|C_{\delta}(y)\right|}{\left|G\left(b\left(t_{j-J}\right), t_{j-J}\right)\right|}<\frac{\mathrm{e}^{\alpha B(T-\widetilde{T})}\left(C_{0}+C_{1}(T-\widetilde{T})\right)}{\inf _{\tilde{T} \leq t \leq T}|G(b(t), t)|}<\infty
$$

for sufficiently small $\delta>0$. Thus, we can apply Lemmas 3.4, 3.5, and 3.6(i) to establish (3.22) and (3.23) for $i=j+1$. (In view of Remark 3.6, recall that $C_{0}$ has been chosen to satisfy (3.28) and note that the bound $\exp [\alpha B(T-\widetilde{T})]\left\{C_{0}+C_{1}(T-\widetilde{T})\right\} / \inf _{\widetilde{T} \leq t \leq T}|G(b(t), t)|$ does not depend on $j$ so that the same $\delta_{1}$ (referred to in Remark 3.6) can be chosen for all $j$.) Finally, making use of Lemma 3.7 and noting, by the induction hypothesis, that $\sup _{x} \mathrm{e}^{-\alpha\left(x-b\left(t_{j}\right)\right)^{-}}\left|v_{\delta}\left(x, t_{j}\right)-v\left(x, t_{j}\right)\right| \leq \mathrm{e}^{\alpha B \delta j}\left(C_{0} \delta+C_{1} \delta^{2} j\right)$, we can proceed as in (3.26) and (3.27) with $T=t_{0}$ and $t_{1}$ replaced by $t_{j}$ and $t_{j+1}$ to establish (3.24) for $i=j+1$.

\subsection{The Chernoff-Petkau method for symmetric Bernoulli walks}

In the backward induction scheme (3.3)-(3.4) considered in Theorem 3.1, the search for the optimal stopping boundary $b_{\delta}\left(t_{i}\right)$ is carried out over the interval $b_{\delta}\left(t_{i-1}\right) \pm \sqrt{\delta}|\log \delta|$, for $i=1, \ldots, K_{\delta}$. In practice, however, the backward induction procedure (3.3) to compute $v_{\delta}\left(x, t_{i}\right)$ is usually carried out over a fixed grid of points instead of within the entire interval. Particularly popular is the symmetric Bernoulli walk approximation with the set of grid points $L=\left\{\beta_{\delta}+\sqrt{\delta} k: k=0, \pm 1, \ldots\right\}$, for which at any time $t_{i}$ every $x \in L$ is characterized as either a stopping point if $g\left(x, t_{i}\right) \geq \mathrm{E} v_{\delta}\left(x+\sqrt{\delta} X, t_{i-1}\right)$ or a continuation point if $\mathrm{E} v_{\delta}(x+$ $\left.\sqrt{\delta} X, t_{i-1}\right)>g\left(x, t_{i}\right)$, where $X$ is a symmetric Bernoulli random variable. A simple way to approximate the optimal stopping boundary $b_{\delta}\left(t_{i}\right)$ is to let

$$
x_{1}=\inf \left\{x \in L: g\left(x, t_{i}\right) \geq \operatorname{E} v_{\delta}\left(x+\sqrt{\delta} X, t_{i-1}\right)\right\}
$$

and $x_{0}=x_{1}-\sqrt{\delta}$, so that $b_{\delta}\left(t_{i}\right)$ is approximated by the value of $x$ satisfying $\Delta\left(x_{1}, t_{i}\right)\left(x-x_{0}\right)+$ $\Delta\left(x_{0}, t_{i}\right)\left(x_{1}-x\right)=0$, where $\Delta\left(x, t_{i}\right)=\frac{1}{2}\left[v_{\delta}\left(x+\sqrt{\delta}, t_{i-1}\right)+v_{\delta}\left(x-\sqrt{\delta}, t_{i-1}\right)\right]-g\left(x, t_{i}\right)$. Note that $\Delta\left(x_{1}, t_{i}\right) \leq 0 \leq \Delta\left(x_{0}, t_{i}\right)$.

Besides this linear interpolation method, Chernoff and Petkau [14], [15] also introduced an extrapolation method which does not explicitly estimate $b_{\delta}\left(t_{i}\right)$. This extrapolation method uses (3.9) to arrive at a system of equations from which the continuous-time optimal stopping boundary $b\left(t_{i}\right)$ is computed. Specifically, with the $(y, s)$ scale as defined in Lemma 3.6, Chernoff and Petkau [15] argued that for large negative $s$ and bounded $y<-\rho$, the $V_{\delta}(y, s)$ in Lemma 3.6(i) can be expressed as $V_{\delta}(y, s)=y^{2}-s-\mathrm{E}\left(-\rho+R_{-\rho-y}\right)^{2}+o(1)$ with $\rho=0.5$ and $R_{z}=1+[z]-z$ for the symmetric Bernoulli case. Since $y^{2}-\mathrm{E}\left(-0.5+R_{-0.5-y}\right)^{2}=-h_{1}(y)$ for $y<0.5$, where

$$
h_{1}(y)=\left[-y^{2}+\inf _{i}(y+i)^{2}\right] \mathbf{1}_{\{y<0.5\}},
$$

it follows that, for a continuation point $\left(x, t_{i}\right)$ in the vicinity of a boundary point $\left(x^{*}, t^{*}\right)$, with $0>x-x^{*}=O(\sqrt{\delta})$ and such that $\left(t_{i}-t^{*}\right) / \delta$ is negatively large but $b\left(t_{i}\right)-x^{*}=$ $b\left(t_{i}\right)-b\left(t^{*}\right)=o(\sqrt{\delta})$,

$$
D\left(x, t_{i}\right):=v_{\delta}\left(x, t_{i}\right)-g\left(x, t_{i}\right) \approx a \delta h_{1}\left(\frac{x-x^{*}}{\sqrt{\delta}}\right) \approx a \delta h_{1}\left(\frac{x-b\left(t_{i}\right)}{\sqrt{\delta}}\right),
$$


where $a=G\left(x^{*}, t^{*}\right)<0$. Recalling that $x_{0}=x_{1}-\sqrt{\delta}$ and that $x_{1}$ is the smallest stopping point on the grid at time $t=t_{i}$, let $D_{0}$ and $D_{1}$ be the values $D\left(x_{0}, t_{i}\right)$ and $D\left(x_{0}-\sqrt{\delta}, t_{i}\right)$, respectively. Then $b_{\delta}\left(t_{i}\right)$ is between $x_{0}$ and $x_{1}$. Since $b_{\delta}(t)=b(t)-0.5 \sqrt{\delta}+o(\sqrt{\delta})$, which implies that (approximately) $b(t)-0.5 \sqrt{\delta}<x_{1}<b(t)+0.5 \sqrt{\delta}$, we use the representation

$$
x_{0}=x_{1}-\sqrt{\delta}=b(t)+v \sqrt{\delta}, \quad x_{0}-\sqrt{\delta}=b(t)+(v-1) \sqrt{\delta},
$$

with $-1.5<v \leq-0.5$, to yield, via (3.30),

$$
D_{0} \approx a \delta h_{1}(v)=a \delta(2 v+1), \quad D_{1} \approx a \delta h_{1}(v-1)=a \delta(4 v) .
$$

The solution to the above linear system is $v=D_{1} /\left(4 D_{0}-2 D_{1}\right)$, which then leads to the following continuity correction for the approximation of $b\left(t_{i}\right)$ by the stopping point $x_{1}$ defined in (3.28):

$$
b\left(t_{i}\right)=x_{0}+\left(\frac{D_{1}}{2 D_{1}-4 D_{0}}\right) \sqrt{\delta} .
$$

Chernoff and Petkau [14], [15] found the corrected Bernoulli-walk approximation (3.31) to work well when applied to a number of well-studied optimal stopping problems. In Section 5 we apply the approximation to a stochastic control problem with an explicit solution and thereby illustrate its accuracy. The function $h_{1}(y)$ in (3.29) first arose in [13, p. 882] as the limit of $-V_{1}(y,-n)+n$ as $n \rightarrow \infty$, where $V_{1}(y,-n)$ is the value function for the symmetric Bernoulli case $(\delta=1)$ with payoff (1.1). Since the payoffs (1.1) and (3.14) differ by $r_{\delta}(y, s)$ defined in (3.15) that is vanishingly small (see (A.2)-(A.6) in Appendix A), we can use the same arguments as those in the proof of Theorem 3.1 to show that the approximation (3.31) still has the $o(\sqrt{\delta})$ error.

\section{Applications to optimal stopping for $S_{\boldsymbol{n}} / \boldsymbol{n}$ and Shepp's urn}

With the payoff function $g(x, t)=x / t$, the classical $S_{n} / n$ problem is to find, for a given initial point $\left(x_{0}, t_{0}\right)$, a stopping time $\tau$ taking values in $\{0,1, \ldots\}$ so as to maximize $\mathrm{E}\left[\left(x_{0}+\right.\right.$ $\left.\left.S_{\tau}\right) /\left(t_{0}+\tau\right)\right]$, where $S_{n}=X_{1}+\cdots+X_{n}, n=1,2, \ldots$, and $X, X_{1}, X_{2}, \ldots$ are i.i.d. with mean 0 and variance 1; see [17] and [19] which prove that it is optimal to stop at the first $n$ at which $x_{0}+S_{n} \geq \beta\left(t_{0}+n\right)$ for some function $\beta(t)$ (stopping boundary). Shepp [46] and Walker [50] have independently shown that $\lim _{n \rightarrow \infty} \beta(n) / n^{1 / 2}=c$ and that the corresponding optimal stopping problem for Brownian motion has stopping boundary $b(t)=c t^{1 / 2}$, where $c=0.83992 \ldots$ is the unique root of the equation $c \phi(c)=\left(1-c^{2}\right) \Phi(c)$, in which $\phi$ and $\Phi$ are the standard normal density and distribution function, respectively. Theorem 3.1 suggests the second-order approximation $\beta(n)=c n^{1 / 2}-\rho+o(1)$ as $n \rightarrow \infty$. Lai and Yao [38] proved this for standard normal $X$. They also computed $\beta(n)$ for symmetric Bernoulli $X$, for which $\rho=0.5$, and showed that $c n^{1 / 2}-0.5$ approximates $\beta(n)$ well even for small $n$.

We next consider a closely related optimal stopping problem introduced by Shepp [46]. Given $n \geq m \geq 0$, consider an urn with $n$ balls, $m$ of which have payoff -1 and $n-m$ of which have payoff +1 . A player draws at random from the urn without replacement until he stops; stopping can occur at time 0 (with no draw at all). As noted by Shepp [46, p. 1000], the urn problem is computationally simpler than the infinite-horizon $S_{n} / n$ problem as we can use backward induction to compute the optimal stopping rule. Instead of $m$, it is more convenient to consider the parameter $k=2 m-n=m-(n-m)$, which is the negative total worth of the urn; see [47]. It is also more convenient to extend $k$ to a continuous variable $w \in[-n, n]$. For any $w \in[-n, n]$, define a $(w, n)$ urn as follows. Let $k$ be the unique integer such that 
$k-2<w \leq k$ and $k$ has the same parity as $n$, so $|k-w-1| \leq 1$. The $(w, n)$ urn consists of $(n+k) / 2-1$ balls with payoff $-1,(n-k) / 2$ balls with payoff 1 , and the remaining ball with payoff $k-w-1$. The expected return under optimal stopping from a $(w, n)$ urn, which will be called its 'maximal expected return', is continuous and nonincreasing in $w$.

Let $\beta(n)$ be the smallest $w$ for which the $(w, n)$ urn has maximal expected return 0 . For a $(\beta(n), n)$ urn, there are two optimal policies; one does not draw at all and the other draws one ball and then proceeds optimally. Both policies yield maximal expected return 0 . Given a $(w, n)$ urn, let $X_{1}^{(n)}, X_{2}^{(n)}, \ldots$ denote the payoffs of the successive draws (without replacement) from the urn and let $S_{j}^{(n)}=X_{1}^{(n)}+\cdots+X_{j}^{(n)}$. An optimal policy is to stop at the first $j$ at which $w+S_{j}^{(n)} \geq \beta(n-j)$. Let $u=n^{-1 / 2} w$ (fixed) and $W_{n}(j / n)=n^{-1 / 2} S_{j}^{(n)}, j=$ $0,1, \ldots$ The optimal policy maximizes $\mathrm{E} W_{n}\left(\tau_{n}\right)$ over stopping times $\tau_{n}$ that take values in $\{0,1 / n, \ldots, 1\}$. As $n \rightarrow \infty, W_{n}$ converges weakly to Brownian bridge $\{W(t), 0 \leq t \leq 1\}$ with $W(1)=-u$. Shepp [46] showed that $\sup _{\tau} \mathrm{E} W(\tau)$ is attained by the stopping time $\tau=\inf \{0 \leq t \leq 1: u+W(t) \geq b(1-t)\}$, where $b(t)=c t^{1 / 2}$ is the stopping boundary of the optimal stopping problem for Brownian motion in the first paragraph of this section. This in turn yields the approximation $\beta(n(1-t)) / n^{1 / 2} \approx b(1-t)$ for $t \in\{0,1 / n, \ldots, 1\}$ and, in particular, $\beta(n)=(c+o(1)) n^{1 / 2}$, as pointed out by Shepp [46] for a slightly different sequence $\bar{\beta}(n)$, where $\bar{\beta}(n)$ is the smallest $k$ for which an urn with $n$ balls and total worth $-k$ has maximal expected return 0 . Note that $\bar{\beta}(n)-2<\beta(n) \leq \bar{\beta}(n)$.

For a $(w, n)$ urn with large $n$ and $w$ close to $c n^{1 / 2}$ (i.e. $(w, n)$ is near the stopping boundary), any fixed number of successive payoffs $X_{j}^{(n)}$ sampled from the $(w, n)$ urn are approximately independent symmetric Bernoulli random variables. The proof of Theorem 3.1 can be modified to extend the results to $X_{j}^{(n)}$ that are asymptotically independent and uniformly bounded, with $\mathrm{E} X_{j}^{(n)}=0$ and $\operatorname{var}\left(X_{j}^{(n)}\right)=1$. Formal application of Theorem 3.1 then yields $\beta(n) / n^{1 / 2}=$ $c-0.5 n^{-1 / 2}+o\left(n^{-1 / 2}\right)$. Simons [47] proposed to use $c n^{1 / 2}-0.5$ as an approximation to $\bar{\beta}(n)$ and conjectured, based on an extensive numerical study, that, as $N \rightarrow \infty, \#\{n \leq N: \bar{\beta}(n)-2<$ $\left.c n^{1 / 2} \leq \bar{\beta}(n)\right\} / N$ and $\#\left\{n \leq N: \bar{\beta}(n)-2<c n^{1 / 2}-0.5 \leq \bar{\beta}(n)\right\} / N$ tend to 0.75 and 1 , respectively. Note that this conjecture is a consequence of $\beta(n)=c n^{1 / 2}-0.5+o(1)$, since the sequence $\left\{c n^{1 / 2}, n=1,2, \ldots\right\}$ is uniformly distributed modulo 1 by Fejér's theorem [31, p. 23]. A technical difficulty in applying Theorem 3.1 to prove this is that the derivative of $b(t)=c t^{1 / 2}$ becomes infinite as $t \rightarrow 0$, and we have not been able to provide a rigorous proof at this point. However, we have carried out an extensive numerical study of the accuracy of $c n^{1 / 2}-0.5$ as an approximation to $\beta(n)$. The results show that this second-order approximation is accurate even for small $n$. A small subset of these results is given in Table 1 .

TABLE 1: Optimal boundary and its second-order approximation for Shepp's urn problem.

\begin{tabular}{lcccccccccc}
\hline$n$ & 1 & 2 & 3 & 4 & 6 & 8 & 10 & 12 & 14 & \multicolumn{1}{c}{16} \\
\hline$\beta(n)$ & 0.000 & 0.500 & 1.000 & 1.000 & 1.500 & 1.894 & 2.153 & 2.370 & 2.586 & 2.797 \\
$c n^{1 / 2}-0.5$ & 0.340 & 0.688 & 0.955 & 1.180 & 1.557 & 1.876 & 2.156 & 2.410 & 2.643 & 2.860 \\
difference & -0.340 & -0.188 & 0.045 & -0.180 & -0.057 & 0.018 & -0.003 & -0.040 & -0.057 & -0.063 \\
\hline$n$ & 18 & 20 & 22 & 24 & 25 & 26 & 27 & 28 & 29 & 30 \\
\hline$\beta(n)$ & 2.998 & 3.206 & 3.408 & 3.603 & 3.655 & 3.791 & 3.820 & 3.973 & 3.977 & 4.114 \\
$c n^{1 / 2}-0.5$ & 3.063 & 3.256 & 3.440 & 3.615 & 3.700 & 3.783 & 3.864 & 3.944 & 4.023 & 4.100 \\
difference & -0.065 & -0.050 & -0.032 & -0.012 & -0.045 & 0.008 & -0.044 & 0.029 & -0.046 & 0.014 \\
\hline
\end{tabular}




\section{Boundary correction in singular stochastic control}

In this section we show how the second-order boundary correction of Section 3 can be extended to compute the optimal boundary in singular stochastic control problems, for which the process does not stop when it hits the boundary but is brought back to the no-action region by the control. Specifically, the control problem is to minimize

$$
\mathrm{E}\left[\int_{0}^{T} h\left(X_{t}, t\right) \mathrm{d} t+\int_{[0, T)} f(t)\left|\mathrm{d} \xi_{t}\right|+k\left(X_{T}\right)\right]
$$

over bounded variation processes $\left\{\xi_{t}\right\}$ that are adapted to the filtration generated by a standard Brownian motion $\left\{B_{t}\right\}$ such that $X_{t}=x+B_{t}+\xi_{t}$; see [2] and [3]. In (5.1) the functions $h$, $f$, and $k$ respectively represent the deviation of $X_{t}$ from 0 , the unit cost of effort $\xi_{t}$, and the terminal penalty when the state is away from the target state 0 . In addition, $h(\cdot, t)$ and $k(\cdot)$ are assumed to be even, convex functions and $f$ to be nonnegative. Expressing $\xi_{t}$ as the difference $\xi_{t}^{+}-\xi_{t}^{-}$, where $\left\{\xi_{t}^{+}\right\}$and $\left\{\xi_{t}^{-}\right\}$are nonnegative, nondecreasing in $t$, the so-called monotone follower problem is to minimize (5.1) subject to the constraint $\xi_{t}^{+} \equiv 0$, which can be solved via a related optimal stopping problem of the form

$$
\min _{\tau \in \mathcal{T}[0, T]} \mathrm{E}\left[\int_{0}^{\tau} h_{x}\left(x+B_{t}, t\right) \mathrm{d} t+f(\tau) \mathbf{1}_{\{\tau<T\}}+k^{\prime}\left(x+B_{T}\right) \mathbf{1}_{\{\tau=T\}}\right],
$$

where $\mathcal{T}[0, T]$ is the class of stopping times taking values in $[0, T]$; see [34].

The special case of the monotone follower problem with $h(x, t)=x^{2}, f \equiv 0$, and $k \equiv 0$, has been solved explicitly by Benes et al. [5], who showed that the optimal (nondecreasing) process $\left\{\xi_{t}^{*}\right\}$ is singular with respect to the Lebesgue measure. It is characterized as the local time of the controlled process $X_{t}^{*}=x+B_{t}-\xi_{t}^{*}$ at the boundary $\partial D=\{(x, t): 0 \leq t \leq T, x=d \sqrt{T-t}\}$, where $d=0.63883 \ldots$ is the solution of a transcendental equation. The optimal control $\left\{\xi_{t}^{*}\right\}$ is applied at the boundary to keep $X_{t}^{*}$ within $D$, which corresponds to the continuation region of the optimal stopping problem (5.2). If $x \in D^{c}$ at time 0 , then $\xi_{0}^{*}$ is such that $X_{0}^{*}$ is the closest point on $\partial D$ to which $x$ is moved instantaneously. Let $U(x, T)$ denote the value function. In this special case, (5.2) reduces to the optimal stopping problem

$$
V(x, T)=\inf _{\tau \in \mathcal{T}[0, T]} \mathrm{E}\left[\int_{0}^{\tau} 2\left(x+B_{t}\right) \mathrm{d} t\right] .
$$

The optimal boundary for the monotone follower problem with value function $U$ is the same as the optimal stopping boundary associated with the value function $V$; moreover, $U_{x}=V$. This connection dates back to Bather and Chernoff [4] and has led to many generalizations and an extensive literature; see [2] and [3] and the references therein.

The optimal stopping problem (5.3) is not of the form (2.1) because the payoff has to be integrated over time up to $\tau$, instead of at the stopping time $\tau$. Conversely, an application of Ito's formula can reduce (5.3) to the form (2.1). More generally, consider the optimal stopping problem (5.2). Let $\kappa(x, t)$ be a solution to the nonhomogeneous heat equation $\partial \kappa / \partial t+\frac{1}{2} \partial^{2} \kappa / \partial x^{2}-h_{x}=0$. Then by Ito's formula we have $\mathrm{E}\left[\kappa\left(x+B_{t}, t\right)-\kappa(x, 0)\right]=$ $\mathrm{E} \int_{0}^{t} h_{x}\left(x+B_{u}, u\right) \mathrm{d} u$. Thus, (5.2) can be expressed in the form (2.1) with $-g(z, t)=$ $f(t) \mathbf{1}_{\{t<T\}}+k^{\prime}(z) \mathbf{1}_{\{t=T\}}+\kappa(z, t)$. In particular, we can write the optimal stopping problem (5.3) as maximizing

$$
-\mathrm{E}\left[\int_{0}^{\tau}\left(x+B_{t}\right) \mathrm{d} t\right]=\mathrm{E}\left[(T-\tau)\left(x+B_{\tau}\right)-T x\right] .
$$


TABLE 2: Uncorrected (U) and corrected (C) random walk approximations against the actual (A) boundary for the monotone follower problem at different values of $\tau=T-t$.

\begin{tabular}{lcccccccccccc}
\hline $10^{3} \tau$ & 2 & 2.5 & 3 & 3.5 & 4 & 5 & 6 & 8 & 10 & 15 & 20 & 30 \\
\hline $10^{2} \mathrm{U}$ & 2.35 & 2.81 & 3.01 & 3.26 & 3.57 & 4.03 & 4.45 & 5.20 & 6.01 & 7.31 & 8.54 & 10.58 \\
$10^{2} \mathrm{C}$ & 2.85 & 3.31 & 3.51 & 3.76 & 4.07 & 4.53 & 4.95 & 5.70 & 6.51 & 7.81 & 9.04 & 11.08 \\
$10^{2} \mathrm{~A}$ & 2.86 & 3.19 & 3.50 & 3.78 & 4.04 & 4.52 & 4.95 & 5.71 & 6.39 & 7.82 & 9.03 & 11.06 \\
\hline
\end{tabular}

Thus, the optimal stopping problem (5.3) associated with the monotone follower case of the control problem (5.1) has a payoff of the form (2.1). We can therefore apply the corrected random walk approximation of Section 3 to compute the optimal reflection boundary. As an illustration, Table 2 (with $\delta=10^{-4}$ and correction started at $t=T-0.0020$ ) shows the dramatic effect of the correction when comparing the corrected Bernoulli walk boundary $(b(t)$ in (3.31) of Section 3.4) and the uncorrected Bernoulli walk boundary $\left(b_{\delta}(t)\right.$ described in Section 3.4) against the actual boundary in the monotone follower problem solved explicitly by Benes et al. [5].

\section{Appendix A}

Proof of Lemma 3.2. Note that $u(y, t)=\mathrm{E}\left[g^{*}\left(y+S_{\sigma(y, t)}\right) \mathbf{1}_{\{\sigma(y, t)=-t\}}-\sigma(y, t)\right]$, where $g^{*}(y)=y^{2} \mathbf{1}_{\{y \leq 0\}}$ and $\sigma(y, t)=\inf \left\{n: 1 \leq n<-t, y+S_{n} \geq z(t+n)\right\}$, inf $\varnothing=-t$. For $t<0$ and $y>z(t)$, suppose that $u(y, t) \geq 0$. Then $\mathrm{P}\left(\sigma(y, t)=-t, y+S_{-t}<0\right)>0$. Moreover, since $z(t)+S_{-t}<y+S_{-t}$,

$$
\begin{aligned}
& \mathrm{E}\left[g^{*}\left(z(t)+S_{\sigma(y, t)}\right) \mathbf{1}_{\{\sigma(y, t)=-t\}}-\sigma(y, t)\right]-u(y, t) \\
& \quad \geq \int_{\left\{\sigma(y, t)=-t, y+S_{-t}<0\right\}}\left[\left(z(t)+S_{-t}\right)^{2}-\left(y+S_{-t}\right)^{2}\right] \mathrm{d} P \\
& \quad>0 .
\end{aligned}
$$

Conversely, $u(z(t), t) \geq \mathrm{E}\left[g^{*}\left(z(t)+S_{\sigma(y, t)}\right) \mathbf{1}_{\{\sigma(y, t)=-t\}}-\sigma(y, t)\right]$ since $u(z(t), t)$ is the optimal expected reward of policies that start at $(z(t), t)$ and take at least one observation. Moreover, $u(z(t), t) \leq \widetilde{V}(z(t), t)=0$. Combining these with (A.1) yields $u(y, t)<0$, contradicting the assumption $u(y, t) \geq 0$.

Since $\widetilde{V}(y+X, t+1)$ is monotone decreasing in $-t$, it follows that $u(y, t)=-1+\mathrm{E} \widetilde{V}(y+$ $X, t+1)$ is monotone decreasing in $-t$. For $y>-\rho=\lim _{s \rightarrow-\infty} z(s), u(y, t)<0$ for all sufficiently large negative $t$, and therefore $\lim _{t \rightarrow-\infty} u(y, t)<0$.

Proof of Lemma 3.4. By the definition of $\mathcal{T}_{\delta, t, y}, y$ is a stopping point if $y \geq|\log \delta|$. While we need consider only $y<|\log \delta|$, most of the inequalities below hold for $|y| \leq \delta^{-\zeta / 2}$ that will be used in the proofs of Lemmas 3.5 and 3.6. In addition to Lemma 3.2, we shall make use of the following uniform bounds for all stopping times $\tau \leq-t \leq \delta^{-\gamma}$ and $|y| \leq \delta^{-\zeta / 2}$.

$$
\begin{gathered}
\delta \mathrm{E}\left(y+S_{\tau}\right)^{2} \leq \delta\left(y^{2}+\delta^{-\gamma}\right) \leq \delta\left(\delta^{-\zeta}+\delta^{-\gamma}\right) \rightarrow 0, \\
\sqrt{\delta} \mathrm{E}\left|y+S_{\tau}\right|^{3} \leq 4 \sqrt{\delta}\left\{|y|^{3}+\delta^{-(3 / 2) \gamma}\right\} \leq 4 \sqrt{\delta}\left(\delta^{-(3 / 2) \zeta}+\delta^{-(3 / 2) \gamma}\right) \rightarrow 0, \\
\delta \mathrm{E}|t+\tau|^{2} \leq \delta^{1-2 \gamma} \rightarrow 0 \\
\mathrm{P}\left(\left|y+S_{-t}\right| \geq \delta^{-1 / 6}\right) \leq \mathrm{P}\left(\max _{n \leq \delta^{-\gamma}}\left|S_{n}\right| \geq \delta^{-1 / 6}-\delta^{-\zeta / 2}\right)=o\left(\delta^{q}\right),
\end{gathered}
$$


for all $q>0$ by Lemma 3.3(ii), recalling (3.2) and that $0<\zeta<\gamma<\frac{1}{3}$; moreover, for $|y| \leq \delta^{-1 / 6}$,

$$
\sqrt{\delta} \mathrm{E}\left[|t+\tau|\left|y+S_{\tau}\right|\right] \leq \delta^{1 / 2-\gamma} \mathrm{E}\left|y+S_{\tau}\right| \leq \delta^{1 / 2-\gamma}\left\{\delta^{-1 / 6}+\delta^{-\gamma / 2}\right\} \rightarrow 0 .
$$

Let $u_{\delta}(y, t)$ be the value of the policy that starts at $(y, t)$, takes one observation, and proceeds optimally thereafter in the optimal stopping problem (3.18). Let $\widetilde{g}(y)=h_{\delta}(y)$ for $|y| \leq \delta^{-1 / 6}$, and $\widetilde{g}(y)=y^{2} \mathbf{1}_{\{y \leq 0\}}$ for $|y|>\delta^{-1 / 6}$. In view of (3.16), $\sup _{y}\left|\widetilde{g}(y)-y^{2} \mathbf{1}_{\{y \leq 0\}}\right|=O(1)$ as $\delta \rightarrow 0$. Therefore, [11, Theorem 4.1], on a modified version of the associated problem holds for general random walks satisfying (3.2) and can be applied to show that, as $t \rightarrow-\infty$,

$$
u_{\delta}(y, t)+t \leq u(y, t)+o(1)+\Delta(y, t) \text { for } \delta^{-\zeta / 2} \geq y>-\rho+\varepsilon,
$$

where $u$ is defined in Lemma 3.2 and

$$
\begin{aligned}
\Delta(y, t):= & \sup _{1 \leq \tau \leq-t} \mathrm{E}\left|r_{\delta}\left(y+S_{\tau}, t+\tau\right)\right| \mathbf{1}_{\{\tau<-t\}} \\
& +\mathrm{E}\left(\left|h_{\delta}\left(y+S_{-t}\right)\right| \mathbf{1}_{\left\{\left|y+S_{-t}\right|>\delta^{-1 / 6}\right\}}+\left(y+S_{-t}\right)^{2} \mathbf{1}_{\left\{y+S_{-t}<-\delta^{-1 / 6}\right\}}\right) .
\end{aligned}
$$

By (3.15), we have

$$
\begin{array}{rl}
\sup _{1 \leq \tau \leq-t} & \mathrm{E}\left|r_{\delta}\left(y+S_{\tau}, t+\tau\right)\right| \mathbf{1}_{\{\tau<-t\}} \\
\leq & A \sup _{1 \leq \tau \leq-t} \mathrm{E}\left[\delta\left(y+S_{\tau}\right)^{2}+\sqrt{\delta}\left|y+S_{\tau}\right|^{3}+\sqrt{\delta}|t+\tau|\left|y+S_{\tau}\right|\right] \\
& +\sum_{n=1}^{-t-1} \mathrm{E}\left|r_{\delta}\left(y+S_{n}, t+n\right)\right| \mathbf{1}_{\left\{\left|y+S_{n}\right|>\delta^{-1 / 6}\right\}} .
\end{array}
$$

From (3.15)-(3.17), (A.2)-(A.6), and Hölder's inequality, it follows that

$$
\sup _{-\rho+\varepsilon<y \leq \delta^{-\zeta / 2},-\delta^{-\gamma} \leq t \leq-1} \Delta(y, t) \rightarrow 0 \quad \text { as } \delta \rightarrow 0 .
$$

It then follows, from Lemma 3.2 and (A.7), that if $-\delta^{-\gamma} \leq t \leq-|\log \delta|$, then $(y, t)$ is a stopping point (with payoff $-t+o(1)$ upon stopping in view of (3.14)) for every $y>-\rho+\varepsilon$ and for all sufficiently small $\delta>0$.

Proof of Lemma 3.5. If $y<-|\log \delta|$, then $(y, t)$ is a continuation point, in view of the definition of $\mathcal{T}_{\delta, t, y}$. For $-|\log \delta| \leq y<-\rho-\varepsilon$ and $-\delta^{-\gamma} \leq t \leq-|\log \delta|$, to show that $(y, t)$ is a continuation point if $\delta$ is sufficiently small, we make use of (A.2)-(A.6) and

$$
\mathrm{E}\left|h_{\delta}\left(y+S_{-t}\right)\right| \mathbf{1}_{\left\{\left|y+S_{-t}\right|>\delta^{-1 / 6}\right\}}+\sum_{n=1}^{-t-1} \mathrm{E}\left|r_{\delta}\left(y+S_{n}, t+n\right)\right| \mathbf{1}_{\left\{\left|y+S_{n}\right|>\delta^{-1 / 6}\right\}} \rightarrow 0,
$$

which follows from (3.17), (A.5), and Hölder's inequality. It then suffices to show that $\widetilde{V}(y):=$ $\lim _{t \rightarrow-\infty} \widetilde{V}(y, t)>0$ if $y<-\rho-\varepsilon$, recalling that $\widetilde{V}(y, t)$ is monotone decreasing in $y$ and in $-t$; see (3.19) and the paragraph preceding Lemma 3.2. Letting $\tau(y, t)=(-t) \wedge \inf \{n: y+$ $\left.S_{n}>-\rho\right\}$, we can apply Doob's optional stopping theorem as in (3.4) and (3.5) of [13] to obtain

$$
\begin{aligned}
\mathrm{E} g\left(y+S_{\tau(y, t)}, t+\tau(y, t)\right)= & y^{2}-t-E\left(y+S_{\tau(y, t)}\right)^{2} \mathbf{1}_{\{\tau(y, t)<-t\}} \\
& -\mathrm{E}\left(y+S_{\tau(y, t)}\right)^{2} \mathbf{1}_{\left\{\tau(y, t)=-t, y+S_{\tau(y, t)} \geq 0\right\}} .
\end{aligned}
$$


Fix $y<-\rho-\varepsilon$ and let $c=-\rho-y$. Note that, as $-t \rightarrow \infty$,

$$
\begin{gathered}
\mathrm{E}\left(y+S_{\tau(y, t)}\right)^{2} \mathbf{1}_{\{\tau(y, t)<-t\}}=\mathrm{E}\left(-\rho+R_{c}\right)^{2}+o(1)=\rho^{2}-2 \rho \mathrm{E} R_{c}+\mathrm{E} R_{c}^{2}+o(1), \\
\mathrm{E}\left(y+S_{\tau(y, t)}\right)^{2} \mathbf{1}_{\left\{\tau(y, t)=-t, y+S_{\tau(y, t)} \geq 0\right\}}=\mathrm{E}\left(-\rho+R_{c}\right)^{2} \mathbf{1}_{\left\{-\rho+R_{c} \geq 0, \tau(y, t)=-t\right\}} \rightarrow 0,
\end{gathered}
$$

by the uniform integrability of $R_{c}^{2}$ (cf. [32]). Moreover, $y^{2}=(\rho+c)^{2}=\rho^{2}+2 \rho c+c^{2}$. Putting this in (A.8) and applying (A.9) and (A.10) then yields

$$
\mathrm{E} g\left(y+S_{\tau(y, t)}, t+\tau(y, t)\right)=-t+2 \rho\left(c+\mathrm{E} R_{c}\right)-\mathrm{E} R_{c}^{2}+c^{2}+o(1)
$$

as $-t \rightarrow \infty$. From this and Lemma 3.1, $\widetilde{V}(y, t) \geq \operatorname{Eg}\left(y+S_{\tau(y, t)}, t+\tau(y, t)\right)+t \geq c^{2}+o(1)$.

Proof of Lemma 3.6. Taylor expansion of $g-\pi_{g}$ around $\left(x^{*}, t^{*}\right)$ yields, for $t<t^{*}$,

$$
\left(g-\pi_{g}\right)(x, t)=G\left(x^{*}, t^{*}\right)\left(t-t^{*}\right)+O\left(\left|t-t^{*}\right|^{2}+\left|x-x^{*}\right|^{3}+\left|t-t^{*}\right|\left|x-x^{*}\right|\right) .
$$

We can apply Lemma 2.1 to conclude that $\partial^{2} v / \partial x^{2}$ and $\partial^{2} v / \partial x \partial t=-\frac{1}{2} \partial^{3} v / \partial x^{3}$ are continuous in the continuation region up to the boundary $b(\cdot)$; moreover, $\frac{1}{2}\left(\partial^{2} / \partial x^{2}\right)(v-g)=-G$ at $x=b(t)$. Note that $v\left(x, t^{*}\right)=g\left(x, t^{*}\right)$ for $x \geq x^{*}$ and that $\left(\partial^{2} / \partial x^{2}\right) v\left(x, t^{*}\right)$ has a discontinuity at $x=x^{*}$. A Taylor expansion around $x^{*}$ yields

$$
\left(v-\pi_{g}\right)\left(x, t^{*}\right)=-G\left(x^{*}, t^{*}\right)\left(x-x^{*}\right)^{2} \mathbf{1}_{\left\{x \leq x^{*}\right\}}+O\left(\left|x-x^{*}\right|^{3}\right) .
$$

In view of (3.5), $-G\left(x^{*}, t^{*}\right)=\left|G\left(x^{*}, t^{*}\right)\right|>0$. From (A.11) and (A.12), it follows that $g_{\delta}$ satisfies (3.14)-(3.16) for $-\delta^{-\gamma} \leq s \leq 0$, for every choice of $0<\gamma<\frac{1}{3}$. To prove that (3.17) holds (with $h_{\delta}$ thus defined) for this range of $\gamma$ and any $q \geq p>1$ and $0<\zeta<\gamma$, it suffices to show that

$$
\begin{gathered}
\sup _{\left|x-x^{*}\right| \leq \delta^{(1-\zeta) / 2}, t^{*}-\delta^{1-\gamma} \leq t \leq t^{*}, n \leq \delta^{-\gamma}} \mathrm{E}\left|g\left(x+\sqrt{\delta} S_{n}, t\right)\right|^{p}=O(1), \\
\sup _{\left|x-x^{*}\right| \leq \delta^{(1-\zeta) / 2}, n \leq \delta^{-\gamma}} \mathrm{E}\left|v\left(x+\sqrt{\delta} S_{n}, t^{*}\right)\right|^{p}=O(1) .
\end{gathered}
$$

From (2.3), (3.7), and (3.2), (A.13) follows, noting that, for some large constant $M>0$,

$$
\mathrm{E}\left|g\left(x+\sqrt{\delta} S_{n}, t\right)\right|^{p} \leq M \mathrm{e}^{M p\left|x+\sqrt{\delta} S_{n}\right|} \leq M \mathrm{e}^{M p|x|}\left\{\mathrm{E}^{M p \sqrt{\delta} S_{n}}+\mathrm{Ee}^{-M p \sqrt{\delta} S_{n}}\right\}=O(1) .
$$

Similarly, as $\sup _{x<b\left(t^{*}\right)} \mathrm{e}^{-\alpha^{\prime \prime \prime}\left|x-b\left(t^{*}\right)\right|}\left|v\left(x, t^{*}\right)\right|<\infty$ by Lemma 3.7 and since $v\left(x, t^{*}\right)=$ $g\left(x, t^{*}\right)$ for $x \geq b\left(t^{*}\right)$, (A.14) follows from (3.7) and (3.2).

To prove part (i), recall that $\delta^{-\zeta}=o\left(\delta^{-\gamma}\right)$ and that $\sup _{\widetilde{T} \leq t \leq T^{*}, x \geq b(t)}|g(x, t)| \mathrm{e}^{-\beta(x)}<\infty$ by (3.7) with $\beta(x)=O(x)$ as $x \rightarrow \infty$, and apply Lemmas 3.3(i), 3.4, and 3.5 together with (A.2)-(A.6), (A.8)-(A.10), (3.20), and (3.2). To prove part (ii), let $V^{(c)}(y, s)$ denote the discrete time optimal value function with respect to the payoff function $-s \mathbf{1}_{\{s<0\}}+(c+$ $\left.y^{2} \mathbf{1}_{\{y \leq 0\}}\right) \mathbf{1}_{\{s=0\}}$, which reduces to (1.1) when $c=0$. By [11, Theorem 4.1], (applicable to random walks satisfying (3.2)) together with (A.2)-(A.6) and (3.15)-(3.17), it follows that, as $\delta \rightarrow 0, V^{(-A)}(y, s)+o(1) \leq V_{\delta}(y, s) \leq V^{(A)}(y, s)+o(1)$ uniformly in $-\delta^{-\gamma} \leq s<0$ and $|y| \leq \delta^{-\zeta / 2}$. Observing that $\left|V^{(c)}(y, s)-V^{(0)}(y, s)\right| \leq|c|$, part (ii) then follows from Lemma A.1, below.

Lemma A.1. Let $V^{(0)}(y, s)$ denote the discrete time optimal value function for Chernoff's associated stopping problem (1.1). Then we have $0 \leq y^{2} \mathbf{1}_{\{y \leq 0\}}-s-V^{(0)}(y, s) \leq \rho^{2}+$ $4 \mathrm{E} S_{\tau+}^{3} / 3 \mathrm{E} S_{\tau+}$ for $s=-1,-2, \ldots$ and $-\infty<y<\infty$. 
Proof. Hogan [32] has shown that there exists a nondecreasing optimal stopping boundary $0>z(-1) \geq \cdots \geq-\rho$. The lemma holds trivially for $(y, s)$ with $y \geq z(s)$. For $(y, s)$ with $y<z(s)$, we have, as in (A.8), $V^{(0)}(y, s)=y^{2}-s-\mathrm{E}\left(y+S_{\tau}\right)^{2} \mathbf{1}_{\{\tau<-s\}}-\mathrm{E}(y+$ $\left.S_{\tau}\right)^{2} \mathbf{1}_{\left\{\tau=-s, y+S_{\tau} \geq 0\right\}}$, where $\tau=(-s) \wedge \inf \left\{n: y+S_{n}>z(s+n)\right\}$ is the optimal stopping time. Letting $\tau(c)=\inf \left\{n: S_{n}>c\right\}$ and noting that $0>z(-1) \geq z(-2) \geq \cdots \geq-\rho$, we have $\left(y+S_{\tau}\right)^{2} \mathbf{1}_{\{\tau<-s\}}+\left(y+S_{\tau}\right)^{2} \mathbf{1}_{\left\{\tau=-s, y+S_{\tau} \geq 0\right\}} \leq \rho^{2}+\left(y+S_{\tau(-y)}\right)^{2}$, which together with (3.10) proves the lemma.

\section{Acknowledgements}

The first author gratefully acknowledges support from the National Science Foundation under grant DMS-0305749. The second author gratefully acknowledges support by the National Science Council of Taiwan, ROC.

\section{References}

[1] AitSahlia, F. and Lai, T. L. (2001). Exercise boundaries and efficient approximations to American option prices and hedge parameters. J. Comput. Finance 4, 85-103.

[2] Alvarez, L. H. R. (2001). Singular stochastic control, linear diffusions, and optimal stopping: a class of solvable problems. SIAM J. Control Optimization 39, 1697-1710.

[3] Baldursson, F. M. (1987). Singular stochastic control and optimal stopping. Stochastics 21, 1-40.

[4] Bather, J. And Chernoff, H. (1967). Sequential decisions in the control of a spaceship. In Proc. Fifth Berkeley Symp. Math. Statist. Prob., Vol. III, University of California Press, Berkeley, pp. 181-207.

[5] Benes, V. E., Shepp, L. A. and Witsenhausen, H. S. (1980). Some solvable stochastic control problems. Stochastics 4, 39-83.

[6] Brezzi, M. and LaI, T. L. (2002). Optimal learning and experimentation in bandit problems. J. Econom. Dynamics Control 27, 87-108.

[7] Broadie, M., Glasserman, P. and Kou, S. (1997). A continuity correction for discrete barrier options. Math. Finance 7, 325-348.

[8] Carr, P., Jarrow, R. And Myneni, R. (1992). Alternative characterizations of American put options. Math. Finance 2, 87-106.

[9] Chen, X. And Chadam, J. (2007). A mathematical analysis of the optimal exercise boundary for American put options. SIAM J. Math. Anal. 38, 1613-1641.

[10] Chen, X., Chadam, J., Jiang, L. And Zhang, W. (2007). Convexity of the exercise boundary of the American put option on a zero dividend asset. To appear in Math. Finance.

[11] Chernoff, H. (1965). Sequential tests for the mean of a normal distribution. IV. (Discrete case). Ann. Math. Statist. 36, 55-68.

[12] Chernoff, H. (1972). Sequential Analysis and Optimal Design. Society for Industrial and Applied Mathematics, Philadelphia, PA.

[13] Chernoff, H. and Petkau, A. J. (1976). An optimal stopping problem for sums of dichotomous random variables. Ann. Prob. 4, 875-889.

[14] Chernoff, H. and Petkau, A. J. (1984). Numerical methods for Bayes sequential decision problems. Tech. Rep. ONR 34, MIT Statistics Center.

[15] Chernoff, H. and PetKau, A. J. (1986). Numerical solutions for Bayes sequential decision problems. SIAM J. Sci. Statist. Comput. 7, 46-59.

[16] Chow, Y. S. AND LaI, T. L. (1979). Moments of ladder variables for driftless random walks. Z. Wahrscheinlichkeitsth 48, 253-257.

[17] Chow, Y. S. And Robbins, H. (1965). On optimal stopping rules for $S_{n} / n$. Illinois J. Math. 9, 444-454.

[18] Cox, J., Ross, S. And Rubinstein, M. (1979). Option pricing: a simplified approach. J. Financial Econom. 7, 229-263.

[19] Dvoretzky, A. (1967). Existence and properties of certain optimal stopping rules. In Proc. Fifth Berkeley Symp. Math. Statist. Prob., Vol. I, University of California Press, Berkeley, pp. 441-452.

[20] Eкsтröm, E. (2004). Convexity of the optimal stopping boundary for the American put option. J. Math. Anal. Appl. 299, 147-156.

[21] Ekström, E. (2004). Properties of American option prices. Stoch. Process. Appl. 114, 265-278.

[22] Evans, J. D., Kuske, R. AND Keller, J. B. (2002). American options on assets with dividends near expiry. Math. Finance 12, 219-237. 
[23] Fakeev, A. G. (1970). Optimal stopping rules for stochastic processes with continuous time. Theory Prob. Appl. 15, 324-331.

[24] Feller, W. (1966). An Introduction to Probability Theory and Its Applications, Vol. 2. John Wiley, New York.

[25] Friedman, A. (1979). Optimal stopping problems in stochastic control. SIAM Rev. 21, 71-80.

[26] Friedman, A. (1979). Time dependent free boundary problems. SIAM Rev. 21, 213-221.

[27] Friedman, A. (1981). Variational inequalities in sequential analysis. SIAM J. Math. Anal. 12, 385-397.

[28] Friedman, A. (1982). Asymptotic behavior of the free boundary of parabolic variational inequalities and applications to sequential analysis. Illinois J. Math. 26, 653-697.

[29] Friedman, A. (1982). Variational Principles and Free Boundary Problems. John Wiley, New York.

[30] Gut, A. (1974). On the moments and limit distributions of some first passage times. Ann. Prob. 2, $277-308$.

[31] Hlawka, E. (1984). The Theory of Uniform Distribution. A B Academic Publishers, Berkhamsted.

[32] Hogan, M. (1986). Comments on a problem of Chernoff and Petkau. Ann. Prob. 14, 1058-1063.

[33] JACKA, S. D. (1991). Optimal stopping and the American put. Math. Finance 1, 1-14.

[34] Karatzas, I. and Shreve, S. E. (1984). Connections between optimal stopping and singular stochastic control. I. Monotone follower problems. SIAM J. Control Optimization 22, 856-877.

[35] Karatzas, I. and Shreve, S. E. (1998). Methods of Mathematical Finance. Springer, New York.

[36] Krylov, N. V. (1980). Controlled Diffusion Processes. Springer, New York.

[37] LAI, T. L. (1976). Uniform Tauberian theorems and their applications to renewal theory and first passage problems. Ann. Prob. 4, 628-643.

[38] LaI, T. L. AND YaO, Y. -C. (2006). The optimal stopping problem for $S_{n} / n$ and its ramifications. In Random Walk, Sequential Analysis and Related Topics, eds A. C. Hsiung, Z. Ying and C.-H. Zhang, World Scientific, Singapore, pp. 131-149.

[39] Lamberton, D. (1998). Error estimates for the binomial approximation of American put options. Ann. Appl. Prob. 8, 206-233.

[40] Lamberton, D. (2002). Brownian optimal stopping and random walks. Appl. Math. Optimization 45, $283-324$.

[41] Lorden, G. (1970). On excess over the boundary. Ann. Math. Statist. 41, 520-527.

[42] MCKeAn, H. P., JR. (1965). A free boundary problem for the heat equation arising from a problem in mathematical economics. Industrial Management Rev. 6, 32-39.

[43] Myneni, R. (1992). The pricing of the American option. Ann. Appl. Prob. 2, 1-23.

[44] Peskir, G. (2005). On the American option problem. Math. Finance 15, 169-181.

[45] Peskir, G. And Shiryaev, A. (2006). Optimal Stopping and Free Boundary Problems. Birkhäuser, Basel.

[46] Shepr, L. A. (1969). Explicit solutions to some problems of optimal stopping. Ann. Math. Statist. 40, 993-1010.

[47] Simons, G. (1987). Easily determining which urns are 'favorable'. Statist. Prob. Lett. 5, 43-48.

[48] VAn MoerbeKe, P. (1974). An optimal stopping problem with linear reward. Acta Math. 132, 111-151.

[49] Van Moerbeke, P. (1976). On optimal stopping and free boundary problems. Arch. Ration. Mech. Anal. 60, $101-148$.

[50] Walker, L. H. (1969). Regarding stopping rules for Brownian motion and random walks. Bull. Amer. Math. Soc. 75, 46-50. 